OPEN ACCESS

Edited by:

Ingrid Melle,

University of Oslo, Norway

Reviewed by:

Emma Barkus,

Northumbria University,

United Kingdom

Massimo Tusconi,

University of Cagliari, Italy

*Correspondence:

Stella G. Giakoumaki

sgiakoumaki@uoc.gr

Specialty section: This article was submitted to Schizophrenia,

a section of the journal

Frontiers in Psychiatry

Received: 01 October 2020 Accepted: 08 December 2020

Published: 08 January 2021

Citation:

Karamaouna P, Zouraraki $C$ and Giakoumaki SG (2021) Cognitive

Functioning and Schizotypy: A

Four-Years Study.

Front. Psychiatry 11:613015.

doi: 10.3389/fpsyt.2020.613015

\section{Cognitive Functioning and Schizotypy: A Four-Years Study}

\author{
Penny Karamaouna ${ }^{1,2}$, Chrysoula Zouraraki ${ }^{1,2}$ and Stella G. Giakoumaki ${ }^{1,2 *}$ \\ ${ }^{1}$ Laboratory of Neuropsychology, Department of Psychology, Faculty of Social Sciences, University of Crete, Rethymno, \\ Greece, ${ }^{2}$ University of Crete Research Center for the Humanities, The Social and Educational Sciences (UCRC), University of \\ Crete, Rethymno, Greece
}

Although there is ample evidence from cross-sectional studies indicating cognitive deficits in high schizotypal individuals that resemble the cognitive profile of schizophrenia-spectrum patients, there is still lack of evidence by longitudinal/follow-up studies. The present study included assessments of schizotypal traits and a wide range of cognitive functions at two time points (baseline and 4-years assessments) in order to examine (a) their stability over time, (b) the predictive value of baseline schizotypy on cognition at follow-up and (c) differences in cognition between the two time points in high negative schizotypal and control individuals. Only high negative schizotypal individuals were compared with controls due to the limited number of participants falling in the other schizotypal groups at follow-up. Seventy participants (mean age: 36.17; $70 \%$ females) were assessed at baseline and follow-up. Schizotypal traits were evaluated with the Schizotypal Personality Questionnaire. We found that schizotypal traits decreased over time, except in a sub-group of participants ("schizotypy congruent") that includes individuals who consistently meet normative criteria of inclusion in either a schizotypal or control group. In these individuals, negative schizotypy and aspects of cognitive-perceptual and disorganized schizotypy remained stable. The stability of cognitive functioning also varied over time: response inhibition, aspects of cued attention switching, set-shifting and phonemic/semantic verbal fluency improved at follow-up. High negative schizotypy at baseline predicted poorer response inhibition and semantic switching at follow-up while high disorganized schizotypy predicted poorer semantic processing and complex processing speed/set-shifting. The between-group analyses revealed that response inhibition, set-shifting and complex processing speed/set-shifting were poorer in negative schizotypals compared with controls at both time points, while maintaining set and semantic switching were poorer only at follow-up. Taken together, the findings show differential stability of the schizotypal traits over time and indicate that different aspects of schizotypy predict a different pattern of neuropsychological task performance during a 4-years time window. These results are of significant use in the formulation of targeted early-intervention strategies for high-risk populations.

Keywords: cognitive functioning, follow-up, schizophrenia-spectrum, schizotypal traits, stability over time 


\section{INTRODUCTION}

Paul Meehl and Gordon Claridge have formulated the two dominant theories in the conceptualization of schizotypy, the quasi-dimensional and the fully-dimensional models, respectively. Meehl (1) proposed that schizotypy refers to a genetically mediated personality profile indicating liability to schizophrenia; when risk-factors for the development of the disease co-occur in individuals with elevated schizotypy, a percentage converts into the disease state. Claridge (2) pays particular attention to individual differences in schizotypal characteristics and considers those as traits lying in a continuum; only when exceeding a critical threshold, schizotypal traits indicate liability to schizophrenia, otherwise remaining part of what he describes as "...normal individual variation" [(2), p. 193]. Other reports also quite early suggested that schizotypy indicates proneness to schizophrenia (3) and schizophreniaspectrum personality disorders (4). Since these early appraisals, (a) aspects of schizotypy have consistently been linked with higher rates of schizophrenia-spectrum traits and/or symptoms (5-7), (b) schizotypal personality has been further established as a key-element of the at risk mental state both in healthy high schizotypal individuals (8) and in individuals at clinical high risk for psychosis (9), and (c) the connection between schizotypy and schizophrenia-spectrum disorders was established over the years at several endophenotypic levels (10-17). Most importantly, high schizotypy has been associated with transition into schizophrenia (18-22).

Schizophrenia is a complex disorder characterized by neuroanatomical $(23,24)$, genetic $(25,26)$, psychophysiological $(27,28)$ and functional $(29,30)$ impairments. Among these deficits, cognitive decline is a core feature of the disorder as evidenced by neuropsychological $(31,32)$ as well as functional neuroimaging $(33,34)$ studies. It is of note that cognitive decline is apparent as early as in the prodromal state $(35,36)$ and in the first episode $(33,37)$ of the disorder as well as in individuals at clinical $(38,39)$ or familial $(38,40)$ high-risk. To further strengthen the link between schizophrenia and schizotypy, poor cognitive functioning has also been well-established in high schizotypal individuals [for reviews and a meta-analysis see $(10,14,41)]$, although the severity of cognitive decline in this population is lower compared with schizophrenia patients (41). Nevertheless, non-significant findings on the relationship between schizotypal traits and cognition have also been reported for several neurocognitive domains, such as set-shifting (42-45), problem solving $(46)$, verbal fluency $(46,47)$, working memory (48), verbal memory $(43,47-50)$, processing speed $(46,51)$, and aspects of attention $(50,52)$.

In a high percentage of studies, cognitive impairments are reported to remain stable over time in schizophrenic examinees $(53,54)$. The temporal stability of schizotypy has been demonstrated with confirmatory factor analysis (55). In the same line, schizotypal traits assessed with various versions of the Schizotypal Personality Questionnaire [SPQ; (56)] have been reported (a) to have moderate stability estimates from early adolescence to early adulthood $(57,58)$, with genetic factors explaining a significant percentage of the reported stability (57), (b) to either decrease, remain stable or increase over a 2 years period depending on their baseline levels in young adults (59), (c) to follow a different trajectory over a 2 -years period as negative schizotypy was more stable compared with positive schizotypy in young army conscripts (60) and (d) to remain stable over a 10-years period in psychotic patients and their unaffected siblings, except for disorganized schizotypy which decreased at the 10-years follow up assessment (61). Studies with the Chapman Psychosis Proneness Scales (62-64) in healthy young adults revealed a decrease in physical anhedonia, perceptual aberration and magical thinking at an 18-months (65) and a 2years follow up assessment (66) compared with baseline along with moderate interclass correlation coefficients of all scores (66). Despite the evidence for stability of schizotypal traits over time, evidence on the association of schizotypy with cognitive functioning in longitudinal or follow-up studies is still limited. To our knowledge, only two studies have thus far dealt with the issue. Thus, (a) Wang et al. (67) reported moderate stability of prospective and working memory deficits in a college sample scoring in the top $10 \%$ of the total score in the SPQ over a period of 6 months and (b) Cohen et al. (68) found that the cognitive performance of community participants with increased social anhedonia improved over a 3-years period, except for verbal or visual working memory and attentional vigilance, for which there was no change between the baseline and the followup assessments.

In a previous study (69) we examined a wide range of cognitive functions in a large community sample stratified for schizotypal traits, as assessed with the detailed four-factor model of schizotypy (70). The present study aimed to examine (a) the stability of cognitive task performance and schizotypal traits over a 4-years period, (b) the predictive value of baseline schizotypy on cognition after 4 years of the initial assessment of participants and (c) potential differences in cognitive functioning between two time points (i.e., baseline and follow-up assessment after 4 years) in high schizotypal individuals, as defined with the fourfactor model (i.e., high paranoid or negative or disorganized or cognitive-perceptual or control individuals). Due to lack of participants fulfilling the criteria in order to be included in any schizotypal group, the latter aim was limited only to the comparison between high negative schizotypals and controls.

\section{MATERIALS AND METHODS}

\section{Participants}

A total of 263 participants who had taken part in the Prefrontally Mediated Endophenotypes in the Schizophrenia Spectrum (PreMES) study were contacted for the 4-years followup assessment. Of those, 193 either declined to participate for several reasons or could not be traced (Supplementary Table 1). Therefore, 70 healthy community participants (mean age \pm SD: $36.17 \pm 9.38 ; 21$ males/49 females) were assessed both at baseline and follow-up. They were older, had more years of education and comprised fewer females (all $p$-values <0.05) compared with the drop-out group; there were no differences between participants in the follow-up assessment and those who dropped-out in any SPQ measure except for ideas of 
reference that were slightly higher in the individuals who dropped-out (Supplementary Table 2). Exclusion criteria were as per Karagiannopoulou et al. (69) while a medical history was taken again at follow-up to confirm that there was no health-related change in the participants. The medical history questionnaire was identical at baseline and follow-up assessments and comprised questions related to general health issues [e.g., "Have you visited a medical doctor for any reason during the last five (at baseline)/four (at follow-up) years?" "Are you taking any prescribed or over the counter medication now?" "Are you taking any substances that could be considered addictive, except nicotine and caffeine?"] and questions related specifically to mental health [e.g., "Have you visited a psychiatrist during the last five (at baseline)/four (at follow-up) years?"; Have you visited a psychologist during the last five (at baseline)/four (at follow-up) years, even for counseling required to resolve everyday problems?"; "Do you have any relatives suffering from a psychiatric/psychological disorder?"]; none of the included participants responded positively in any question both at baseline and follow-up. At baseline assessment, participants had been divided into schizotypal groups according to standard criteria (i.e., schizotypy scores falling in the upper $10 \%$ for only one schizotypal factor) derived by a normative sample in Greece (70). In detail, (a) negative schizotypals were those with scores $\geq 18$ in the negative schizotypy factor but with scores $<14$ in the paranoid, $<7$ in the cognitive-perceptual and $<8$ in the disorganized schizotypy factors; (b) paranoid schizotypals were those with scores $\geq 14$ in paranoid schizotypy factor but with scores $<18$ in the negative, $<7$ in the cognitiveperceptual and $<8$ in the disorganized schizotypy factors; (c) cognitive-perceptual schizotypals were those with scores $\geq 7$ in the cognitive-perceptual schizotypy factor but with scores $<18$ in the negative, $<14$ in the paranoid and $<8$ in the disorganized schizotypy factors; (d) disorganized schizotypals were those with scores $\geq 8$ in the disorganized schizotypy factor but with scores $<18$ in the negative, $<14$ in the paranoid, and $<7$ in the cognitive-perceptual schizotypy factors; (e) controls were those who did not meet the criteria for any schizotypy factor.

At follow-up, participants were also administered the SPQ (56) and the above-mentioned criteria were applied for their categorization into schizotypal groups a second time. Of the 70 tested volunteers, 54 fell in the same group (31 controls, two cognitive-perceptual, 20 negative, and one disorganized schizotypals- "schizotypy congruent") and another 16 "converted" into another group (Supplementary Table 3).

The study was approved by the Research Ethics Committee of the University of Crete (approval number: 4/2018/19-032018) and the Bureau for the Protection of Personal Data of the Greek State (approval number: $\Gamma \mathrm{N} / \mathrm{E} \Xi / 749-1 / 21-12-2011$ and $\Gamma N / E \Xi / 2029-1 / 10-11-2016)$. Following oral presentation of the study's aims and methods, participants received written detailed information and gave written informed consent prior to participation.

\section{Assessment of Schizotypy}

Schizotypal traits were evaluated with the Greek version (70) of the SPQ (56). The SPQ is a 74-dichotomous-item questionnaire and items are grouped into nine subscales (ideas of reference, social anxiety, odd beliefs/magical thinking, unusual perceptual experiences, eccentric/odd behavior, lack of close friends, odd speech, constricted affect, and suspiciousness) in analogy to the diagnostic criteria for Schizotypal Personality Disorder (SPD). The subscales are organized into four schizotypal factors (70): negative (including suspiciousness, social anxiety, lack of close friends and constricted affect), paranoid (including ideas of reference, suspiciousness and social anxiety), cognitiveperceptual (including odd beliefs/magical thinking and unusual perceptual experiences), and disorganized (including eccentric/odd behavior and odd speech).

\section{Neuropsychological Assessment Wisconsin Card Sorting Test [WCST; (71)]}

Set-shifting was assessed with a computerized version of the WCST. The task consisted of four stimulus cards that varied along three dimensions (shape, color, and number) and a target card. Participants were asked to match the target card with one if the stimulus cards and feedback was provided after each selection. In the first part of the task, the first match was always scored as correct and the rule applied by the participant was the first sorting principle. After six consecutive correct responses, the sorting principle changed and participants were informed of this. The next match according to either of the two remaining sorting principles was scored as correct and as previously, after six consecutive correct responses, the sorting principle changed again and participants were informed. The third match was scored as correct only when the last sorting principle was applied. In the second part of the task, participants were required to repeat the three sorting principles in the same order. The task was discontinued when six categories were completed or when the target cards were exhausted. Outcome variables were (a) total number of completed categories, (b) number of unrelated matched cards, (c) perseverative errors either of Milner- or Nelson-type [Milner-type perseverative errors: responses that were correct on the immediately preceding stage of the test (72); Nelson-type perseverative errors: all other perseverative errors (71)], and (d) Milner- and Nelson-non perseverative errors.

\section{Letter-Number Sequencing [LNS; (73)]}

Executive working memory was examined with the LNS. The Greek version (74) of the task was used. Strings of increasing difficulty comprising intermingled letters and numbers were read to the participants who were required to recite these strings after reordering of the information (i.e., in numeric and alphabetical order). The outcome variable was the total number of correct responses.

\section{Trail-Making Test [TMT; (75)]}

Processing speed/set-shifting was evaluated with the Greek version (76) of TMT. The task consisted of two parts: in Part A, participants were required to connect 25 consecutively numbered circles, as quickly as possible. In Part B, participants were required to connect 25 consecutively numbered or lettered circles by alternating between the two sequences. The outcome variables were the seconds required by the participants to complete each part of the test. 


\section{Verbal Fluency Test (77)}

The Greek version of the task was used for the assessment of phonemic and semantic fluency. In the phonemic fluency condition, participants were required to produce in 3 consecutive minutes as many as possible words beginning with the letter $\mathrm{X}$ (chi), S (sigma), and A (alpha) according to standard instructions. In the semantic fluency condition, participants were required to produce in 3 consecutive minutes, according to standard instructions, as many as possible words belonging to each of the following semantic categories: animals, fruit and objects. Outcome variables were (a) correct responses, (b) perseverative errors (i.e., words generated more than once), (c) intrusion errors (i.e., words that did not belong to the category required), (d) clusters (i.e., three or more consecutive words beginning with the same two letters and having the same sound or two consecutive words that differed only in a vowel sound or words that were homophones for the phonemic part; three or more consecutive words belonging to the same semantic sub-category in the semantic part), and (e) switches (i.e., total correct responses minus the number of words related to each cluster plus number of clusters) per sub-task.

\section{Raven's Progressive Matrices (78)}

The task assesses abstract reasoning. It comprised five sets of 12 abstract patterns, each with one missing piece. Participants were required to select the choice that best matched the pattern out of the possible answer choices accompanying every pattern. Items within a set were of increasing difficulty. The outcome variable was the total number of correct selections.

\section{Cambridge Neuropsychological Test Automated Battery (CANTAB) Tasks (79)}

Attention Switch task (AST) assesses cued attentional switching. In every trial, an arrow appears on the left or the right half of the computer screen. A cue presented on the screen indicates whether the participant should make a response about the direction of the arrow or the side of the screen that the arrow was presented. A number of trials includes congruent stimuli (i.e., the arrow is on the right half of the screen and points to the right) whereas another number of trials include incongruent stimuli (i.e., the arrow is on the right half of the screen and points to the left), which require higher cognitive demands. Outcome variables were (a) congruency cost in mean correct responses (i.e., the difference between response latency of congruent vs. incongruent trials), (b) switch cost in mean correct responses (i.e., the difference between response latency of non-switched vs. switched trials), (c) total correct responses in switched and nonswitched trials, respectively, (d) total commission errors (i.e., total number of trials in which the examinee responded either before the end of the window or before the appearance of the stimulus) in switched and non-switched trials, respectively.

Stop-Signal task (SST) examines response inhibition. In every trial, a white ring is presented in the center of the screen. After a delay of $500 \mathrm{~ms}$, a white arrow pointing either to the right or to the left is presented within the ring. In the first part of the task, participants were required to press the right-hand button of a touch pad when the arrow points to the right or the left-hand button of the touch pad, when the arrow points to the left. In the second part of the task (five blocks with 64 trials each), participants were required to do as previously unless they hear an auditory stimulus. When they heard the auditory stimulus, participants were required to give no response. Outcome variables were (a) correct responses in the "stop condition" (i.e., when participants are required to give no response), (b) correct responses in the "go condition" (i.e., when participants are required to press either the right- or the left-hand button of the touch pad according to the direction of the arrow), (c) errors in the "stop condition" (i.e., when participants press the button of the touch pad that does not match the direction of the arrow in trials with the auditory stimulus), (d) errors in the "go condition" (i.e., when participants press the button of the touch pad that does not match the direction of the arrow in trials without the auditory stimulus), and (e) reaction time for correct response in the "go condition."

Stockings of Cambridge [SoC; (80)] examines planning and complex problem solving abilities. Participants were required to compare two different arrangements of "balls" in "socks" (one presented on the top half of the screen, the other at the bottom half) and re-arrange (with the minimum possible number of moves), the balls in the lower half in order to match the target arrangement in the upper half. The problems are of increasing difficulty. Participants were required to plan the complete sequence of moves needed prior to their first move. Outcome variables were (a) number of problems solved correctly with the minimum moves, (b) mean number of moves, (c) mean initial thinking time (i.e., the time taken to organize the solution of the problem prior to execution of the first move), and (d) mean subsequent thinking time (i.e., the time required for the achieving the solution to the problem).

Spatial Working Memory [SWM; (80)] was administered for the assessment of spatial working memory and strategy formation. Participants were required to search through an increasing number of boxes randomly arranged on the screen, until they find a token that, at any one time, is hidden in one of the boxes; upon finding the token, participants were required to place it in a "home area." The key instruction was that once a token has been found within a particular box, that box should never be used again to hide a token. On each trial, every box is used once to hide one token, such that the total number of tokens to be found equals to the number of boxes on the screen. Outcome variables were (a) between errors (i.e., times of re-visiting a box in which a token was previously found), (b) within errors (i.e., times of revisiting a box already found to be empty during the same search), (c) double errors (i.e., errors that can be categorized as both within and between errors), (d) strategy score (i.e., an efficient strategy is to follow a predetermined search sequence, beginning with a specified box and then return to start each new sequence with that same box as soon as a token has been found; a high score indicates poor strategy), and (e) mean search preparation time (i.e., the mean time between searches; for the first search it refers to the time between the on-screen presentation of the problem and the first touch of the examinee and for subsequent searches it refers to the time between the placement of token in the home area and the time of the next touch). 


\section{Assessment of Subjective Mood and Feelings on the Day of Testing}

Upon arrival at the laboratory, subjects self-rated their mood and feelings on a battery of 16-item visual analog scales [VAS; (81)] of $10 \mathrm{~cm}$ each. The raw values for each item were weighted with their respective factor loading and the weighted values were allocated to "alertness," "anxiety," and "discontentment" factors (82). The outcome variable was the average of the weighted values for each factor.

\section{Statistical Analyses}

The stability of schizotypal traits and neuropsychological task performance between baseline and follow-up assessments was examined with a series of repeated measures analyses of variance (ANOVAs) with time point (two levels) as the withinsubjects factor separately for the total sample $(n=70)$ and the "schizotypy congruent" group $(n=54)$. The rank order stability of participants' schizotypy scores was further examined with Pearson correlations between the baseline and followup scores separately for the total sample and the "schizotypy congruent" group. Associations between schizotypal traits at baseline and neuropsychological task performance at follow-up were examined with a series of stepwise regressions (dependent variable: neuropsychological measure; predictors: schizotypal factor scores; confounders: sex, age and smoking habits at baseline) separately for the whole sample and the "schizotypy congruent" group. To reduce the probability of type I error, we applied a Bonferroni correction (0.05/9 neuropsychological tasks $=0.0056$ ); therefore, only $p$-values $<0.0056$ were considered as significant and $p$-values $<0.05$ were considered as trends for significance. In the "schizotypy congruent" sub-sample, betweengroup differences between controls $(n=31)$ and negative schizotypals $(n=20)$ in demographic variables (age, years of education, smoking habits), VAS and SPQ scores at followup were examined with parametric or non-parametric tests according to normality of the distribution; sex differences were examined with $\chi^{2}$ analysis and differences in neuropsychological tasks performance were examined with $2 \times 2$ repeated measures ANOVAs with time point (baseline and follow-up) as the withinand group (control or negative schizotypals) as the betweensubjects factors. Due to the small sample size of the two groups, we considered the between-group differences as preliminary findings and we did not apply the Bonferroni correction in these analyses.

\section{RESULTS}

\section{Stability of Schizotypal Traits}

In the analyses of the total sample, there was a significant main effect of time point for the cognitive-perceptual $\left[F_{(1,69)}=15.55\right.$, $\left.p<0.001, \eta^{2}=0.184\right]$, negative $\left[F_{(1,69)}=6.88, p<0.05, \eta^{2}\right.$ $=0.091]$, paranoid $\left[F_{(1,69)}=14.62, p<0.001, \eta^{2}=0.175\right]$, and disorganized $\left[F_{(1,69)}=8.22, p<0.005, \eta^{2}=0.106\right]$ factor scores as well as for the total SPQ score $\left[F_{(1,69)}=19.56, p<\right.$ $\left.0.001, \eta^{2}=0.221\right]$ and the ideas of reference $\left[F_{(1,69)}=6.41, p\right.$ $\left.<0.05, \eta^{2}=0.085\right]$, odd beliefs $\left[F_{(1,69)}=14.42, p<0.001, \eta^{2}=\right.$ $0.173]$, unusual perceptual experiences $\left[F_{(1,69)}=4.59, p<0.05\right.$, $\left.\eta^{2}=0.062\right]$, eccentric behavior $\left[F_{(1,69)}=4.84, p<0.05, \eta^{2}=\right.$ $0.065]$, odd speech $\left[F_{(1,69)}=7.09, p<0.05, \eta^{2}=0.093\right]$, and suspiciousness $\left[F_{(1,69)}=9.11, p<0.005, \eta^{2}=0.117\right]$ sub-scale scores. In all these measures, there was a decrease in the scores at follow-up (Table 1, upper panel). The Pearson's correlations between the factor scores of the two time points revealed that the strongest coefficient was that of negative schizotypy scores $(r=$ 0.907, $p<0.001)$ followed by paranoid $(r=0.658, p<0.001)$, cognitive-perceptual $(r=0.637, p<0.001)$, and disorganized $(r$ $=0.510, p<0.001)$ schizotypy. At a sub-scale level, the strongest correlation was found for lack of close friends $(r=0.819, p<$ $0.001)$ followed by strong coefficients for constricted affect $(r=$ $0.783, p<0.001)$, odd speech $(r=0.730, p<0.001)$, excessive social anxiety $(r=0.695, p<0.001)$, eccentric behavior $(r=$ $0.625, p<0.001)$, and suspiciousness $(r=0.611, p<0.001)$ and moderate coefficients for odd beliefs $(r=0.589, p<0.001)$, ideas of reference $(r=0.487, p<0.001)$, and unusual perceptual experiences $(r=0.465, p<0.001)$. The correlation coefficient for the total SPQ score between baseline and follow-up was $(r=$ $0.771, p<0.001$ ). A detailed description of the correlation matrix is provided in Supplementary Table 4 (upper panel).

In the analyses of the "schizotypy congruent" group, though, a significant main effect of time point was found only for the cognitive-perceptual $\left[F_{(1,53)}=6.93, p<0.05, \eta^{2}=0.116\right]$, paranoid $\left[F_{(1,53)}=4.48, p<0.05, \eta^{2}=0.078\right]$, and disorganized $\left[F_{(1,53)}=5.37, \mathrm{p}<0.05, \eta^{2}=0.092\right]$ factor scores as well as the total SPQ score $\left[F_{(1,53)}=13.04, p<0.001, \eta^{2}=0.198\right]$, the odd beliefs $\left[F_{(1,53)}=5.71, p<0.05, \eta^{2}=0.097\right]$, and eccentric behavior $\left[F_{(1,53)}=7.50, p<0.05, \eta^{2}=0.124\right]$ subscale scores. As previously, the scores at follow-up decreased compared with baseline (Table 1, lower panel). The remaining effects of time point were not significant (all $p$-values $>0.100$ ). The Pearson's correlations between the factor scores of the two time points revealed that the strongest coefficient was that of negative schizotypy scores $(r=0.931, p<0.001)$ followed by cognitive-perceptual $(r=0.705, p<0.001)$, paranoid $(r=0.700$, $p<0.001)$, and disorganized $(r=0.482, p<0.001)$ schizotypy. At a sub-scale level, the strongest correlation was found for lack of close friends $(r=0.837, p<0.001)$ followed by strong coefficients for constricted affect $(r=0.796, p<0.001)$, eccentric behavior $(r=0.758, p<0.001)$, excessive social anxiety $(r=0.718, p<$ $0.001)$, odd speech $(r=0.693, p<0.001)$, suspiciousness $(r=$ $0.630, p<0.001)$, and odd beliefs $(r=0.608, p<0.001)$ and moderate coefficients for ideas of reference $(r=0.460, p<0.001)$ and unusual perceptual experiences $(r=0.457, p<0.001)$. The correlation coefficient for the total SPQ score between baseline and follow-up was $r=0.891$ ( $p<0.001)$. A detailed description of the correlation matrix is provided in Supplementary Table 4 (lower panel).

\section{Stability of Neuropsychological Task Performance}

In the total sample, there was a significant main effect of time point for (a) SST correct responses in the "stop" $\left[F_{(1,69)}=6.28, p\right.$ $\left.<0.05, \eta^{2}=0.083\right]$ and "go" $\left[F_{(1,69)}=5.79, p<0.05, \eta^{2}=0.077\right]$ conditions, errors in the "stop" $\left[F_{(1,69)}=12.53, p<0.001, \eta^{2}=\right.$ 
TABLE 1 | SPQ metrics (mean \pm SD) at baseline and follow-up assessment.

\begin{tabular}{lccc}
\hline WHOLE SAMPLE $(\boldsymbol{N}=\mathbf{7 0})$ & & & \\
\hline & $\begin{array}{c}\text { Baseline } \\
\text { assessment }\end{array}$ & $\begin{array}{c}\text { Follow-up } \\
\text { assessment }\end{array}$ & $\boldsymbol{P}$-value \\
\hline Ideas of reference & $1.69 \pm 1.72$ & $1.21 \pm 1.26$ & $\mathbf{0 . 0 1 4}$ \\
Excessive social anxiety & $2.80 \pm 2.47$ & $2.41 \pm 2.12$ & 0.081 \\
Odd beliefs & $1.81 \pm 1.90$ & $1.09 \pm 1.59$ & $<\mathbf{0 . 0 0 1}$ \\
Unusual perceptual experiences & $1.11 \pm 1.38$ & $0.77 \pm 1.19$ & $\mathbf{0 . 0 3 6}$ \\
Eccentric behavior & $1.39 \pm 1.75$ & $1.00 \pm 1.63$ & $\mathbf{0 . 0 3 1}$ \\
Lack of close friends & $2.77 \pm 2.75$ & $2.73 \pm 2.90$ & 0.834 \\
Odd speech & $2.43 \pm 2.09$ & $1.96 \pm 1.92$ & $\mathbf{0 . 0 1 0}$ \\
Constricted affect & $2.19 \pm 2.16$ & $2.09 \pm 2.39$ & 0.582 \\
Suspiciousness & $2.40 \pm 2.01$ & $1.80 \pm 1.71$ & $\mathbf{0 . 0 0 4}$ \\
Total score & $18.49 \pm 9.98$ & $15.06 \pm 9.00$ & $<\mathbf{0 . 0 0 1}$ \\
Cognitive-perceptual factor score & $2.93 \pm 2.88$ & $1.86 \pm 2.33$ & $<\mathbf{0 . 0 0 1}$ \\
Paranoid factor score & $6.89 \pm 4.14$ & $5.43 \pm 3.39$ & $<\mathbf{0 . 0 0 1}$ \\
Negative factor score & $9.99 \pm 7.01$ & $9.03 \pm 7.11$ & $\mathbf{0 . 0 1 1}$ \\
Disorganized factor score & $4.03 \pm 3.42$ & $2.96 \pm 2.81$ & $\mathbf{0 . 0 0 5}$
\end{tabular}

SCHIZOTYPY CONGRUENT GROUP $(N=54)$

\begin{tabular}{lccc}
\hline Ideas of reference & $1.26 \pm 1.44$ & $1.13 \pm 1.20$ & 0.495 \\
Excessive social anxiety & $2.83 \pm 2.46$ & $2.54 \pm 2.24$ & 0.226 \\
Odd beliefs & $1.50 \pm 1.65$ & $1.04 \pm 1.57$ & $\mathbf{0 . 0 2 0}$ \\
Unusual perceptual experiences & $0.78 \pm 1.09$ & $0.65 \pm 1.01$ & 0.390 \\
Eccentric behavior & $1.31 \pm 1.75$ & $0.89 \pm 1.42$ & $\mathbf{0 . 0 0 8}$ \\
Lack of close friends & $3.17 \pm 2.96$ & $3.06 \pm 3.10$ & 0.640 \\
Odd speech & $2.04 \pm 1.75$ & $1.78 \pm 1.80$ & 0.176 \\
Constricted affect & $2.46 \pm 2.31$ & $2.41 \pm 2.60$ & 0.799 \\
Suspiciousness & $2.26 \pm 2.08$ & $1.87 \pm 1.86$ & 0.100 \\
Total score & $17.67 \pm 10.37$ & $15.35 \pm 9.48$ & $<\mathbf{0 . 0 0 1}$ \\
Cognitive-perceptual factor score & $2.27 \pm 2.23$ & $1.69 \pm 2.06$ & $\mathbf{0 . 0 1 1}$ \\
Paranoid factor score & $6.35 \pm 3.76$ & $5.54 \pm 3.52$ & $\mathbf{0 . 0 3 9}$ \\
Negative factor score & $10.50 \pm 7.45$ & $9.87 \pm 7.66$ & 0.106 \\
Disorganized factor score & $3.63 \pm 3.34$ & $2.67 \pm 2.48$ & $\mathbf{0 . 0 2 4}$ \\
\hline
\end{tabular}

SPQ, Schizotypal Personality Questionnaire.

Significant effects are marked in bold.

$0.154]$ and "go" $\left[F_{(1,69)}=6.67, p<0.05, \eta^{2}=0.088\right]$ conditions and reaction time for correct responses in the "go" condition $\left[F_{(1,69)}=4.01, p<0.05, \eta^{2}=0.055\right]$, (b) AST correct switched responses $\left[F_{(1,69)}=7.27, p<0.05, \eta^{2}=0.097\right]$, (c) WCST Milner perseverative errors $\left[F_{(1,69)}=5.05, p<0.05, \eta^{2}=0.068\right]$ and Nelson non-perseverative-errors $\left[F_{(1,69)}=5.28, p<0.05, \eta^{2}=\right.$ $0.071]$, (d) phonemic fluency correct responses $\left[F_{(1,69)}=17.54, p\right.$ $\left.<0.001, \eta^{2}=0.203\right]$, perseverative errors $\left[F_{(1,69)}=4.45, p<\right.$ $\left.0.05, \eta^{2}=0.061\right]$ and switches $\left[F_{(1,69)}=14.84, p<0.001, \eta^{2}\right.$ $=0.177]$, and (e) semantic fluency correct responses $\left[F_{(1,69)}=\right.$ 13.23, $\left.p<0.001, \eta^{2}=0.161\right]$. In all these measures, performance improved at follow-up compared with baseline (Table 2, upper panel). The remaining effects of time point were not significant (all $p$-values $>0.058$ ).

In the "schizotypy congruent" group, there was a significant main effect of time point for (a) SST correct responses in the "stop" $\left[F_{(1,53)}=14.08, p<0.001, \eta^{2}=0.210\right]$ and "go" $\left[F_{(1,53)}=\right.$ $\left.6.78, p<0.05, \eta^{2}=0.113\right]$ conditions, errors in the "stop" $\left[F_{(1,53)}\right.$ $\left.=14.46, p<0.001, \eta^{2}=0.214\right]$ and "go" $\left[F_{(1,53)}=7.98, p<0.05\right.$, $\left.\eta^{2}=0.131\right]$ conditions and reaction time for correct responses in the "go" condition $\left[F_{(1,53)}=5.83, p<0.05, \eta^{2}=0.099\right]$, (b) AST correct switched responses $\left[F_{(1,53)}=6.22, p<0.05, \eta^{2}=\right.$ 0.107], (d) phonemic fluency correct responses $\left[F_{(1,53)}=13.92\right.$, $\left.p<0.001, \eta^{2}=0.208\right]$ and switches $\left[F_{(1,53)}=14.06, p<0.001\right.$, $\left.\eta^{2}=0.210\right]$, and (e) semantic fluency correct responses $\left[F_{(1,53)}=\right.$ $\left.8.99, p<0.005, \eta^{2}=0.145\right]$. In all these measures, performance improved at follow-up compared with baseline (Table 2, lower panel). The remaining effects of time point were not significant (all $p$-values $>0.066$ ).

\section{Association of Schizotypal Factor Scores at Baseline With Neuropsychological Performance at Follow-Up Stop-Signal Task}

In the whole sample, (a) high paranoid schizotypy along with female sex were associated $\left[F_{(2,69)}=7.52, p<0.001, R^{2}=\right.$ 0.183 ] with fewer correct responses in the "stop condition" (paranoid schizotypy: beta $=-0.344, t=-3.120, p<0.005$ female sex: beta $=0.253, t=2.295, p<0.05$ ), (b) female sex was associated $\left[F_{(1,69)}=13.37, p<0.001, R^{2}=0.164\right]$ with more correct responses (beta $=0.405, t=3.657, p<$ 0.001 ) in the "go condition," (c) high negative schizotypy was associated $\left[F_{(1,69)}=5.78, p<0.005, R^{2}=0.147\right]$ with more errors in the "stop condition" (beta $=0.293, t=2.535, p<0.05$ ). In the "schizotypy congruent" sub-sample, only high negative schizotypy was associated $\left[F_{(1,53)}=7.85, p<0.001, R^{2}=0.235\right]$ with errors in the "stop condition" (beta $=0.392, t=3.113$, $p<0.005)$.

\section{Spatial Working Memory}

In the whole sample, only older age was significantly associated $\left[F_{(1,69)}=9.02, p<0.005, R^{2}=0.117\right]$ with more total between errors (beta $=0.342, t=3.003, p<0.005$ ) and prolonged mean search preparation time $\left[F_{(1,69)}=9.50, p<0.005, R^{2}=0.123\right.$; beta $=0.350, t=3.082, p<0.005]$. In the "schizotypy congruent" group, a similar pattern was observed: older age was significantly associated $\left[F_{(1,53)}=9.93, p<0.005, R^{2}=0.160\right]$ with more total between errors (beta $=0.400, t=3.150, p<0.005$ ) and longer mean search preparation time $\left[F_{(1,53)}=11.85, p<0.001\right.$, $R^{2}=0.186$; beta $\left.=0.431, t=3.442, p<0.001\right]$.

\section{Wisconsin Card Sorting Test}

In the whole sample, only high negative schizotypy tended to be associated $\left[F_{(1,69)}=5.31, p<0.05, R^{2}=0.072\right]$ with fewer completed categories (beta $=-0.269, t=-2.303, p<0.05$ ). In the "schizotypy congruent" group, we did not find any significant models (all $p$-values $>0.05$ ).

\section{Phonemic Verbal Fluency}

In the whole sample, only older age tended to be associated $\left[F_{(1,69)}=5.95, p<0.05, R^{2}=0.284\right]$ with more perseverative errors (beta $=0.284, t=2.439, p<0.05$ ). In the "schizotypy congruent" sub-sample, female sex was (a) associated $\left[F_{(1,53)}=\right.$ $8.69, p<0.005, R^{2}=0.143$ ] with correct responses (beta $=0.378$, 
TABLE 2 | Neuropsychological task performance (mean \pm SD) at baseline and follow-up.

\section{WHOLE SAMPLE $(N=70)$}

$\begin{array}{ccc}\text { Baseline } & \text { Follow-up } & P \text {-value } \\ \text { assessment } & \text { assessment }\end{array}$

\begin{tabular}{lccc}
\hline STOCKINGS OF CAMBRIDGE & & & \\
Problems solved correctly & $9.37 \pm 1.45$ & $9.24 \pm 1.42$ & 0.514 \\
Mean moves & $4.09 \pm 0.36$ & $4.14 \pm 0.41$ & 0.277 \\
Mean initial thinking time & $5611.63 \pm$ & $5048.33 \pm$ & 0.108 \\
& 4316.97 & 2826.96 & \\
Mean subsequent thinking time & $416.62 \pm$ & $342.96 \pm$ & 0.116 \\
& 431.95 & 374.58 & \\
STOP-SIGNAL TASK & & & \\
Correct responses- "stop & $42.27 \pm 5.99$ & $44.17 \pm 6.86$ & $\mathbf{0 . 0 1 5}$ \\
condition" & & & $\mathbf{0 . 0 1 9}$ \\
Correct responses- "go & $238.71 \pm 1.48$ & $239.14 \pm 1.63$ & $\mathbf{0 . 0 1 9}$ \\
condition" & & & $\mathbf{0 . 0 0 1}$ \\
Errors- "stop condition" & $0.53 \pm 0.74$ & $0.23 \pm 0.52$ & $\mathbf{0 . 0 1 2}$ \\
Errors- "go condition" & $1.31 \pm 1.46$ & $0.86 \pm 1.63$ & $\mathbf{0 . 0 4 9}$ \\
RT correct responses-"go & $517.95 \pm$ & $547.21 \pm$ \\
condition" & 124.02 & 144.90 &
\end{tabular}

\section{SPATIAL WORKING MEMORY TASK}

\begin{tabular}{|c|c|c|c|}
\hline Total between-errors & $18.10 \pm 14.69$ & $16.87 \pm 13.16$ & 0.443 \\
\hline Total within-errors & $2.26 \pm 3.22$ & $2.51 \pm 3.64$ & 0.625 \\
\hline Total double-errors & $1.19 \pm 2.35$ & $1.06 \pm 1.71$ & 0.695 \\
\hline Strategy score & $40.97 \pm 5.32$ & $41.54 \pm 5.82$ & 0.409 \\
\hline Mean search preparation time & $\begin{array}{c}1173.79 \pm \\
403.97\end{array}$ & $\begin{array}{c}1098.17 \pm \\
387.58\end{array}$ & 0.058 \\
\hline \multicolumn{4}{|l|}{ ATTENTION SWITCH TASK } \\
\hline $\begin{array}{l}\text { Mean congruency } \\
\text { cost-correct responses }\end{array}$ & $81.28 \pm 54.22$ & $88.93 \pm 63.67$ & 0.377 \\
\hline $\begin{array}{l}\text { Mean switch cost-correct } \\
\text { responses }\end{array}$ & $\begin{array}{c}-108.32 \pm \\
110.67\end{array}$ & $\begin{array}{c}-112.16 \pm \\
84.19\end{array}$ & 0.739 \\
\hline $\begin{array}{l}\text { Total correct switched } \\
\text { responses }\end{array}$ & $77.33 \pm 7.61$ & $79.84 \pm 5.20$ & 0.009 \\
\hline $\begin{array}{l}\text { Total correct non-switched } \\
\text { responses }\end{array}$ & $72.30 \pm 6.45$ & $72.61 \pm 4.57$ & 0.666 \\
\hline $\begin{array}{l}\text { Total commission } \\
\text { errors - switched responses }\end{array}$ & $0.07 \pm 0.31$ & $0.00 \pm 0.00$ & 0.058 \\
\hline $\begin{array}{l}\text { Total commission } \\
\text { errors-non-switched } \\
\text { responses }\end{array}$ & $0.03 \pm 0.17$ & $0.06 \pm 0.24$ & 0.418 \\
\hline \multicolumn{4}{|c|}{ WISCONSIN CARD SORTING TEST } \\
\hline Completed categories & $5.40 \pm 1.12$ & $5.57 \pm 0.91$ & 0.187 \\
\hline Unrelated cards & $1.19 \pm 1.92$ & $0.74 \pm 2.51$ & 0.130 \\
\hline Nelson perseverative errors & $1.96 \pm 2.00$ & $1.57 \pm 1.57$ & 0.147 \\
\hline Milner perseverative errors & $3.23 \pm 2.78$ & $2.34 \pm 2.20$ & 0.028 \\
\hline Nelson non-perseverative errors & $4.60 \pm 3.66$ & $3.50 \pm 3.06$ & 0.025 \\
\hline Milner non-perseverative errors & $3.39 \pm 3.24$ & $2.73 \pm 2.35$ & 0.066 \\
\hline \multicolumn{4}{|l|}{ PHONEMIC VERBAL FLUENCY } \\
\hline Correct responses & $36.99 \pm 8.07$ & $40.33 \pm 9.70$ & $<0.001$ \\
\hline Perseverative errors & $0.74 \pm 1.15$ & $0.46 \pm 0.76$ & 0.038 \\
\hline Intrusion errors & $1.74 \pm 2.21$ & $1.43 \pm 1.55$ & 0.300 \\
\hline Clusters & $2.16 \pm 1.49$ & $2.19 \pm 1.89$ & 0.908 \\
\hline Switches & $32.07 \pm 7.51$ & $35.07 \pm 8.39$ & $<0.001$ \\
\hline
\end{tabular}

TABLE 2 | Continued

WHOLE SAMPLE $(N=70)$

\begin{tabular}{|c|c|c|c|}
\hline & $\begin{array}{c}\text { Baseline } \\
\text { assessment }\end{array}$ & $\begin{array}{l}\text { Follow-up } \\
\text { assessment }\end{array}$ & $P$-value \\
\hline \multicolumn{4}{|l|}{ SEMANTIC VERBAL FLUENCY } \\
\hline Correct responses & $55.07 \pm 13.51$ & $60.63 \pm 13.35$ & $<0.001$ \\
\hline Perseverative errors & $1.20 \pm 1.30$ & $1.01 \pm 1.32$ & 0.363 \\
\hline Intrusion errors & $3.33 \pm 9.23$ & $1.63 \pm 4.58$ & 0.077 \\
\hline Clusters & $7.93 \pm 2.47$ & $8.27 \pm 2.69$ & 0.312 \\
\hline Switches & $32.61 \pm 8.47$ & $34.03 \pm 8.25$ & 0.246 \\
\hline \multicolumn{4}{|l|}{ TRAIL MAKING TEST } \\
\hline Part A & $21.70 \pm 6.58$ & $21.07 \pm 6.80$ & 0.418 \\
\hline Part B & $41.59 \pm 11.56$ & $41.01 \pm 12.77$ & 0.669 \\
\hline \multicolumn{4}{|c|}{ LETTER-NUMBER SEQUENCING } \\
\hline Total correct responses & $11.03 \pm 3.25$ & $11.20 \pm 2.85$ & 0.676 \\
\hline \multicolumn{4}{|c|}{ RAVEN'S PROGRESSIVE MATRICES } \\
\hline Total correct responses & $52.43 \pm 4.73$ & $52.19 \pm 5.80$ & 0.585 \\
\hline \multicolumn{4}{|c|}{ SCHIZOTYPY CONGRUENT GROUP $(N=54)$} \\
\hline & $\begin{array}{c}\text { Baseline } \\
\text { assessment }\end{array}$ & $\begin{array}{l}\text { Follow-up } \\
\text { assessment }\end{array}$ & $P$-value \\
\hline \multicolumn{4}{|l|}{ STOCKINGS OF CAMBRIDGE } \\
\hline Problems solved correctly & $9.39 \pm 1.41$ & $9.17 \pm 1.42$ & 0.301 \\
\hline Mean moves & $4.10 \pm 0.38$ & $4.17 \pm 0.41$ & 0.239 \\
\hline Mean initial thinking time & $\begin{array}{c}5684.15 \pm \\
4399.97\end{array}$ & $\begin{array}{c}5053.24 \pm \\
3019.83\end{array}$ & 0.112 \\
\hline Mean subsequent thinking time & $\begin{array}{c}390.75 \pm \\
407.65\end{array}$ & $\begin{array}{c}360.31 \pm \\
391.39\end{array}$ & 0.569 \\
\hline \multicolumn{4}{|l|}{ STOP-SIGNAL TASK } \\
\hline $\begin{array}{l}\text { Correct responses_-"stop } \\
\text { condition" }\end{array}$ & $42.56 \pm 6.16$ & $45.54 \pm 6.37$ & $<0.001$ \\
\hline $\begin{array}{l}\text { Correct responses - "go } \\
\text { condition" }\end{array}$ & $238.69 \pm 1.56$ & $239.19 \pm 1.59$ & 0.012 \\
\hline Errors - "stop condition" & $0.52 \pm 0.75$ & $0.19 \pm 0.52$ & $<0.001$ \\
\hline Errors - "go condition" & $1.35 \pm 1.54$ & $0.81 \pm 1.59$ & 0.007 \\
\hline $\begin{array}{l}\text { RT correct responses - "go } \\
\text { condition" }\end{array}$ & $\begin{array}{c}523.41 \pm \\
120.82\end{array}$ & $\begin{array}{c}562.60 \pm \\
146.02\end{array}$ & 0.019 \\
\hline \multicolumn{4}{|c|}{ SPATIAL WORKING MEMORY TASK } \\
\hline Total between-errors & $18.22 \pm 15.88$ & $17.43 \pm 13.90$ & 0.684 \\
\hline Total within-errors & $2.44 \pm 3.57$ & $2.52 \pm 3.70$ & 0.908 \\
\hline Total double-errors & $1.30 \pm 2.63$ & $1.13 \pm 1.83$ & 0.687 \\
\hline Strategy score & $40.52 \pm 5.76$ & $41.31 \pm 5.73$ & 0.320 \\
\hline Mean search preparation time & $\begin{array}{c}1186.28 \pm \\
409.65\end{array}$ & $\begin{array}{c}1122.65 \pm \\
421.64\end{array}$ & 0.159 \\
\hline \multicolumn{4}{|l|}{ ATTENTION SWITCH TASK } \\
\hline $\begin{array}{l}\text { Mean congruency } \\
\text { cost-correct responses }\end{array}$ & $85.93 \pm 56.20$ & $89.69 \pm 66.21$ & 0.708 \\
\hline $\begin{array}{l}\text { Mean switch cost-correct } \\
\text { responses }\end{array}$ & $\begin{array}{c}-104.98 \pm \\
104.69\end{array}$ & $\begin{array}{c}-108.22 \pm \\
85.79\end{array}$ & 0.792 \\
\hline $\begin{array}{l}\text { Total correct switched } \\
\text { responses }\end{array}$ & $78.26 \pm 5.85$ & $80.34 \pm 5.27$ & 0.016 \\
\hline $\begin{array}{l}\text { Total correct non-switched } \\
\text { responses }\end{array}$ & $72.79 \pm 5.26$ & $72.91 \pm 4.38$ & 0.886 \\
\hline $\begin{array}{l}\text { Total commission } \\
\text { errors - switched responses }\end{array}$ & $0.08 \pm 0.33$ & $0.00 \pm 0.00$ & 0.103 \\
\hline $\begin{array}{l}\text { Total commission } \\
\text { errors - non-switched } \\
\text { responses }\end{array}$ & $0.02 \pm 0.14$ & $0.04 \pm 0.19$ & 0.569 \\
\hline
\end{tabular}

(Continued) 
TABLE 2 | Continued

\section{SCHIZOTYPY CONGRUENT GROUP $(N=54)$}

\begin{tabular}{|c|c|c|c|}
\hline & $\begin{array}{c}\text { Baseline } \\
\text { assessment }\end{array}$ & $\begin{array}{l}\text { Follow-up } \\
\text { assessment }\end{array}$ & $P$-value \\
\hline \multicolumn{4}{|c|}{ WISCONSIN CARD SORTING TEST } \\
\hline Completed categories & $5.39 \pm 1.09$ & $5.56 \pm 0.92$ & 0.192 \\
\hline Unrelated cards & $1.11 \pm 1.70$ & $0.83 \pm 2.79$ & 0.384 \\
\hline Nelson perseverative errors & $1.91 \pm 1.92$ & $1.56 \pm 1.59$ & 0.236 \\
\hline Milner perseverative errors & $3.07 \pm 2.73$ & $2.31 \pm 2.20$ & 0.115 \\
\hline Nelson non-perseverative errors & $4.50 \pm 3.62$ & $3.50 \pm 3.18$ & 0.071 \\
\hline Milner non-perseverative errors & $3.46 \pm 3.46$ & $2.74 \pm 2.50$ & 0.092 \\
\hline \multicolumn{4}{|l|}{ PHONEMIC VERBAL FLUENCY } \\
\hline Correct responses & $36.20 \pm 8.35$ & $39.48 \pm 9.17$ & $<0.001$ \\
\hline Perseverative errors & $0.76 \pm 1.23$ & $0.46 \pm 0.77$ & 0.066 \\
\hline Intrusion errors & $1.83 \pm 2.38$ & $1.33 \pm 1.58$ & 0.179 \\
\hline Clusters & $2.11 \pm 1.63$ & $1.98 \pm 1.73$ & 0.634 \\
\hline Switches & $31.39 \pm 7.69$ & $34.81 \pm 8.36$ & $<0.001$ \\
\hline \multicolumn{4}{|l|}{ SEMANTIC VERBAL FLUENCY } \\
\hline Correct responses & $55.02 \pm 12.67$ & $60.57 \pm 12.76$ & 0.004 \\
\hline Perseverative errors & $1.13 \pm 1.26$ & $1.04 \pm 1.41$ & 0.684 \\
\hline Intrusion errors & $2.81 \pm 8.40$ & $0.89 \pm 1.99$ & 0.109 \\
\hline Clusters & $8.09 \pm 2.42$ & $8.48 \pm 2.58$ & 0.341 \\
\hline Switches & $32.63 \pm 8.06$ & $32.94 \pm 7.85$ & 0.824 \\
\hline \multicolumn{4}{|l|}{ TRAIL MAKING TEST } \\
\hline Part A & $21.99 \pm 6.33$ & $21.42 \pm 7.02$ & 0.522 \\
\hline Part B & $42.27 \pm 11.19$ & $40.79 \pm 10.98$ & 0.291 \\
\hline \multicolumn{4}{|c|}{ LETTER-NUMBER SEQUENCING } \\
\hline Total correct responses & $10.85 \pm 2.93$ & $10.89 \pm 2.85$ & 0.965 \\
\hline \multicolumn{4}{|c|}{ RAVEN'S PROGRESSIVE MATRICES } \\
\hline Total correct responses & $52.30 \pm 4.72$ & $52.13 \pm 5.67$ & 0.748 \\
\hline
\end{tabular}

$R T$, Reaction time.

Significant effects are marked in bold.

$t=2.948, p<0.005)$ and (b) associated $\left[F_{(1,53)}=9.20, p<\right.$ $\left.0.005, R^{2}=0.150\right]$ with more clusters (beta $=0.388, t=3.033$, $p<0.005)$.

\section{Semantic Verbal Fluency}

In the whole sample, (a) high disorganized schizotypy along with more cigarettes smoked daily were associated $\left[F_{(2,69)}=\right.$ 7.40, $\left.p<0.001, R^{2}=0.181\right]$ with more intrusion errors (disorganized schizotypy: beta $=0.329, t=2.959, p<0.005$; cigarettes: beta $=0.239, t=2.147, p<0.05)$ and $(b)$ high negative schizotypy score was associated $\left[F_{(1,69)}=9.92, p<\right.$ $\left.0.005, R^{2}=0.127\right]$ with fewer switches (beta $=-0.357, t$ $=-3.149, p<0.005)$. High disorganized schizotypy tended to be associated $\left[F_{(1,69)}=4.03, p<0.05, R^{2}=0.056\right]$ with fewer correct responses (beta $=-0.236, t=-2.007, p$ $<0.05)$. In the "schizotypy congruent" group, high negative schizotypy was associated $\left[F_{(1,53)}=12.32, p<0.001, R^{2}=\right.$ 0.192 ] with fewer switches (beta $=-0.438, t=-3.510, p<$ $0.001)$ and tended to be associated $\left[F_{(1,53)}=6.14, p<0.05\right.$,
TABLE 3 | Demographic characteristics, VAS, and SPQ scores (mean \pm SD) of the control and negative schizotypal groups.

\begin{tabular}{|c|c|c|c|}
\hline & $\begin{array}{l}\text { Controls } \\
(n=31)\end{array}$ & $\begin{array}{c}\text { Negative } \\
\text { schizotyplas } \\
(n=20)\end{array}$ & $P$-value \\
\hline Age (years) ${ }^{a}$ & $41.32 \pm 10.22$ & $38.80 \pm 7.52$ & 0.347 \\
\hline Education (years) ${ }^{a}$ & $17.27 \pm 2.05$ & $16.05 \pm 2.63$ & 0.072 \\
\hline Sex (male/female) ${ }^{b}$ & $7 / 24$ & $9 / 11$ & 0.092 \\
\hline Cigarettes/dayc & $3.74 \pm 7.03$ & $3.10 \pm 7.30$ & 0.250 \\
\hline VAS anxiety ${ }^{a}$ & $2.49 \pm 1.79$ & $2.47 \pm 1.52$ & 0.969 \\
\hline VAS discontentment ${ }^{a}$ & $1.48 \pm 1.01$ & $2.00 \pm 1.01$ & 0.083 \\
\hline VAS alertness $^{a}$ & $5.30 \pm 0.75$ & $5.10 \pm 1.03$ & 0.437 \\
\hline SPQ total score ${ }^{a}$ & $8.26 \pm 4.95$ & $25.65 \pm 3.67$ & $<0.001$ \\
\hline SPQ Cognitive-Perceptual factor score ${ }^{c}$ & $1.42 \pm 1.93$ & $1.50 \pm 1.50$ & 0.385 \\
\hline SPQ Paranoid factor score ${ }^{a}$ & $3.81 \pm 2.83$ & $8.10 \pm 3.14$ & $<0.001$ \\
\hline SPQ Negative factor score ${ }^{c}$ & $4.13 \pm 3.05$ & $19.20 \pm 1.06$ & $<0.001$ \\
\hline SPQ Disorganized factor score ${ }^{c}$ & $1.61 \pm 1.36$ & $3.90 \pm 2.63$ & 0.003 \\
\hline
\end{tabular}

a One-way Analysis of variance.

${ }^{b}$ Chi-square analysis.

${ }^{c}$ Mann-Whitney analysis.

$S P Q$, Schizotypal Personality Questionnaire.

VAS, Visual Analog Scales.

Significant between-group differences are marked in bold.

$\left.R^{2}=0.106\right]$ with more intrusion errors (beta $=0.325, t=2.477$, $p<0.05)$.

\section{Trail Making Test}

In the whole sample, (a) older age was associated $\left[F_{(1,69)}=\right.$ 9.38, $\left.p<0.005, R^{2}=0.121\right]$ with prolonged completion time of the first part (beta $=0.348, t=3.062, p<0.005$ ) and (b) high disorganized schizotypy along with high cognitiveperceptual schizotypy and more cigarettes smoked daily were associated $\left[F_{(3,69)}=11.25, p<0.001, R^{2}=0.338\right]$ with prolonged completion time of the second part of the task (disorganized schizotypy: beta $=0.381, t=3.721, p<0.001$; cognitiveperceptual schizotypy: beta $=0.215, t=2.083, p<0.05$; cigarettes: beta $=0.339, t=3.325, p<0.001)$. In the "schizotypy congruent" sub-group, (a) older age was again associated $\left[F_{(1,53)}\right.$ $=9.09, p<0.005, R^{2}=0.149$ ] with prolonged completion time of the first part (beta $=0.386, t=3.016, p<0.005$ ) and (b) only high disorganized schizotypy was associated $\left[F_{(1,53)}\right.$ $\left.=18.60, p<0.001, R^{2}=0.263\right]$ with prolonged completion time of the second part of the task (beta $=0.513, t=4.312$, $p<0.001)$.

\section{Differences Between Controls and Negative Schizotypals} Demographics, VAS, and SPQ Scores

There were no differences in any demographic variables or VAS scores between the control and the negative schizotypal groups (all $p$-values $>0.07$ ). The negative schizotypal group, though, had higher total SPQ, paranoid, negative and disorganized factor scores (all 
TABLE 4 | Neuropsychological task performance (mean \pm SD) of the control and negative schizotypal groups.

\begin{tabular}{|c|c|c|c|c|c|c|c|}
\hline & \multicolumn{2}{|c|}{ Controls $(n=31)$} & \multicolumn{2}{|c|}{ Negative schizotypals $(n=20)$} & \multirow{2}{*}{$\begin{array}{c}P \text {-value } \\
\text { group }\end{array}$} & \multirow{2}{*}{$\begin{array}{c}P \text {-value time } \\
\text { point }\end{array}$} & \multirow{2}{*}{$\begin{array}{c}P \text {-value } \\
\text { group } x \\
\text { time point }\end{array}$} \\
\hline & Baseline & Follow-up & Baseline & Follow-up & & & \\
\hline \multicolumn{8}{|l|}{ STOP-SIGNAL TASK } \\
\hline Correct responses - "stop condition" & $44.45 \pm 6.16$ & $47.10 \pm 5.84$ & $39.80 \pm 5.32$ & $43.70 \pm 6.33$ & 0.009 & $<0.001$ & 0.464 \\
\hline Correct responses - "go condition" & $239.19 \pm 0.98$ & $239.55 \pm 1.09$ & $237.90 \pm 1.92$ & $238.85 \pm 1.66$ & 0.006 & 0.001 & 0.128 \\
\hline Errors - "stop condition" & $0.39 \pm 0.62$ & $0.00 \pm 0.00$ & $0.70 \pm 0.86$ & $0.45 \pm 0.76$ & 0.013 & 0.001 & 0.470 \\
\hline Errors - "go condition" & $0.90 \pm 1.04$ & $0.45 \pm 1.09$ & $2.05 \pm 1.88$ & $1.15 \pm 1.66$ & 0.011 & 0.001 & 0.248 \\
\hline RT correct responses - "go condition" & $558.60 \pm 112.09$ & $590.48 \pm 137.57$ & $469.39 \pm 123.20$ & $530.10 \pm 158.33$ & 0.031 & 0.011 & 0.413 \\
\hline \multicolumn{8}{|l|}{ WISCONSIN CARD SORTING TEST } \\
\hline Completed categories & $5.68 \pm 0.65$ & $5.81 \pm 0.48$ & $4.85 \pm 1.46$ & $5.25 \pm 1.21$ & 0.007 & 0.032 & 0.264 \\
\hline Unrelated cards & $1.13 \pm 1.28$ & $0.26 \pm 0.51$ & $1.20 \pm 2.31$ & $1.85 \pm 4.43$ & 0.159 & 0.739 & 0.025 \\
\hline Nelson perseverative errors & $1.87 \pm 1.48$ & $1.65 \pm 1.43$ & $1.85 \pm 2.54$ & $1.30 \pm 1.78$ & 0.654 & 0.214 & 0.601 \\
\hline Milner perseverative errors & $3.03 \pm 2.56$ & $2.68 \pm 2.29$ & $2.90 \pm 2.99$ & $1.70 \pm 1.98$ & 0.273 & 0.127 & 0.403 \\
\hline Nelson non-perseverative errors & $4.81 \pm 3.47$ & $3.65 \pm 2.82$ & $4.25 \pm 4.04$ & $3.15 \pm 3.57$ & 0.513 & 0.055 & 0.958 \\
\hline Milner non-perseverative errors & $3.65 \pm 2.58$ & $2.61 \pm 1.84$ & $3.50 \pm 4.68$ & $2.75 \pm 3.26$ & 0.996 & 0.046 & 0.747 \\
\hline \multicolumn{8}{|l|}{ SEMANTIC VERBAL FLUENCY } \\
\hline Correct responses & $54.00 \pm 10.74$ & $62.23 \pm 12.60$ & $56.60 \pm 15.05$ & $59.75 \pm 12.83$ & 0.984 & 0.005 & 0.195 \\
\hline Perseverative errors & $1.13 \pm 1.09$ & $1.16 \pm 1.46$ & $1.15 \pm 1.57$ & $0.65 \pm 0.81$ & 0.394 & 0.310 & 0.249 \\
\hline Intrusion errors & $2.35 \pm 7.47$ & $0.42 \pm 0.72$ & $3.90 \pm 10.30$ & $1.65 \pm 3.01$ & 0.276 & 0.112 & 0.904 \\
\hline Clusters & $8.00 \pm 2.14$ & $8.48 \pm 2.84$ & $8.15 \pm 2.91$ & $8.50 \pm 2.35$ & 0.889 & 0.349 & 0.880 \\
\hline Switches & $32.74 \pm 7.39$ & $36.06 \pm 7.81$ & $33.10 \pm 9.29$ & $29.20 \pm 5.89$ & 0.055 & 0.843 & 0.016 \\
\hline \multicolumn{8}{|l|}{ TRAIL MAKING TEST } \\
\hline Part A & $22.85 \pm 6.86$ & $22.44 \pm 8.20$ & $20.32 \pm 5.27$ & $19.30 \pm 4.75$ & 0.095 & 0.458 & 0.754 \\
\hline Part B & $40.39 \pm 9.07$ & $36.54 \pm 8.26$ & $45.45 \pm 13.56$ & $45.03 \pm 10.56$ & 0.012 & 0.116 & 0.206 \\
\hline
\end{tabular}

Significant effects are marked in bold.

RT, Reaction Time.

$p$-values $<0.005)$. A detailed description is provided in Table 3.

\section{Neuropsychological Task Performance}

The descriptives of the two groups' performance in the tasks with either between-group differences or interactions involving group are presented in Table 4; the descriptives of the remaining tasks are presented in Supplementary Table 5.

\section{Stop Signal Task}

Significant main effects of group and time point were found for the correct responses in the "stop" [group: $F_{(1,49)}=7.43, p<$ $0.01 ; \eta^{2}=0.132$; time point: $F_{(1,49)}=14.81, p<0.001 ; \eta^{2}=$ 0.232 ] and "go" [group: $F_{(1,49)}=8.27, p<0.01 ; \eta^{2}=0.144$; time point: $\left.F_{(1,49)}=11.52, p<0.001 ; \eta^{2}=0.190\right]$ conditions, in the errors made in the "stop" [group: $F_{(1,49)}=6.68, p<0.05 ; \eta^{2}=$ 0.120 ; time point: $F_{(1,49)}=11.46, p<0.001 ; \eta^{2}=0.189$ ] and "go" [group: $F_{(1,49)}=7.04, p<0.05 ; \eta^{2}=0.126$; time point: $F_{(1,49)}$ $\left.=12.44, p<0.001 ; \eta^{2}=0.202\right]$ conditions and in the reaction time for correct responses [group: $F_{(1,49)}=4.95, p<0.05 ; \eta^{2}$ $=0.092$; time point: $\left.F_{(1,49)}=7.04, p<0.05 ; \eta^{2}=0.126\right]$ in the "go" condition. Overall, (a) the negative schizotypal group gave fewer correct responses, made more errors in both conditions and had prolonged reaction time in the correct responses of the "go" condition compared with the control group and (b) both groups gave more correct responses, made fewer errors and had longer reaction time at follow-up compared with the baseline assessment.

\section{Wisconsin Card Sorting Test}

The negative schizotypal group completed fewer categories [main effect of group: $\left.F_{(1,49)}=8.06, p<0.01 ; \eta^{2}=0.141\right]$ compared with the control group. Both groups completed more categories and made fewer Milner non-perseverative errors at follow-up compared with the baseline assessment [main effect of time point for completed categories: $F_{(1,49)}=4.86, p<0.05 ; \eta^{2}=0.090$; main effect of time point for Milner non-perseverative errors: $\left.F_{(1,49)}=4.20, p<0.05 ; \eta^{2}=0.079\right]$. We also found a significant group $\times$ time point interaction for unrelated cards $\left[F_{(1,49)}=\right.$ 5.34, $p<0.05 ; \eta^{2}=0.098$; the negative schizotypal group selected more unrelated cards at follow-up compared with the baseline assessment, while the opposite pattern was observed in the control group (Figure 1, upper panel). No other significant main effects or interactions were found (all $p$-values $>0.055$ ).

\section{Trail Making Test}

The negative schizotypal group had poorer performance in the second part of the task at both time points [main effect of group: 


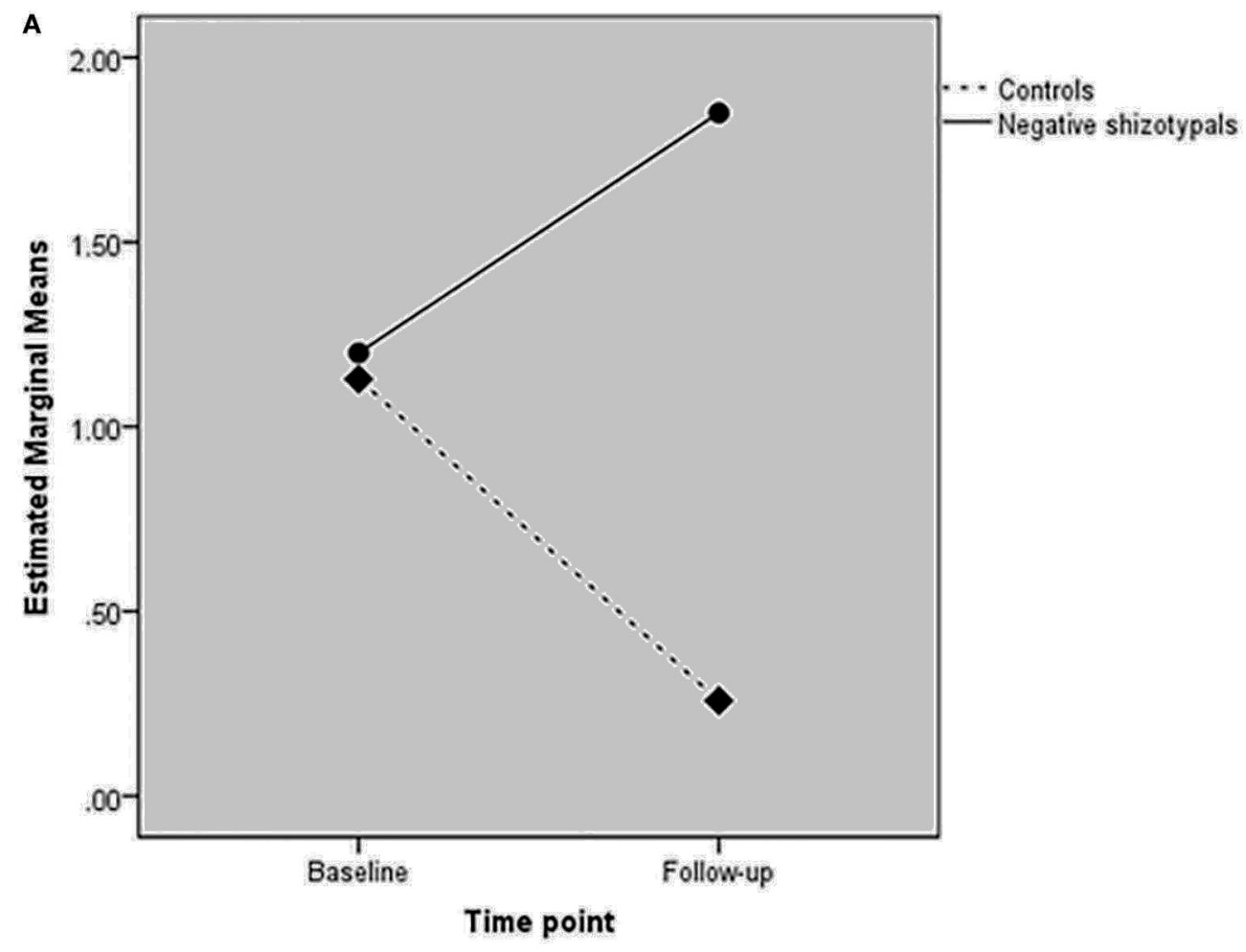

B

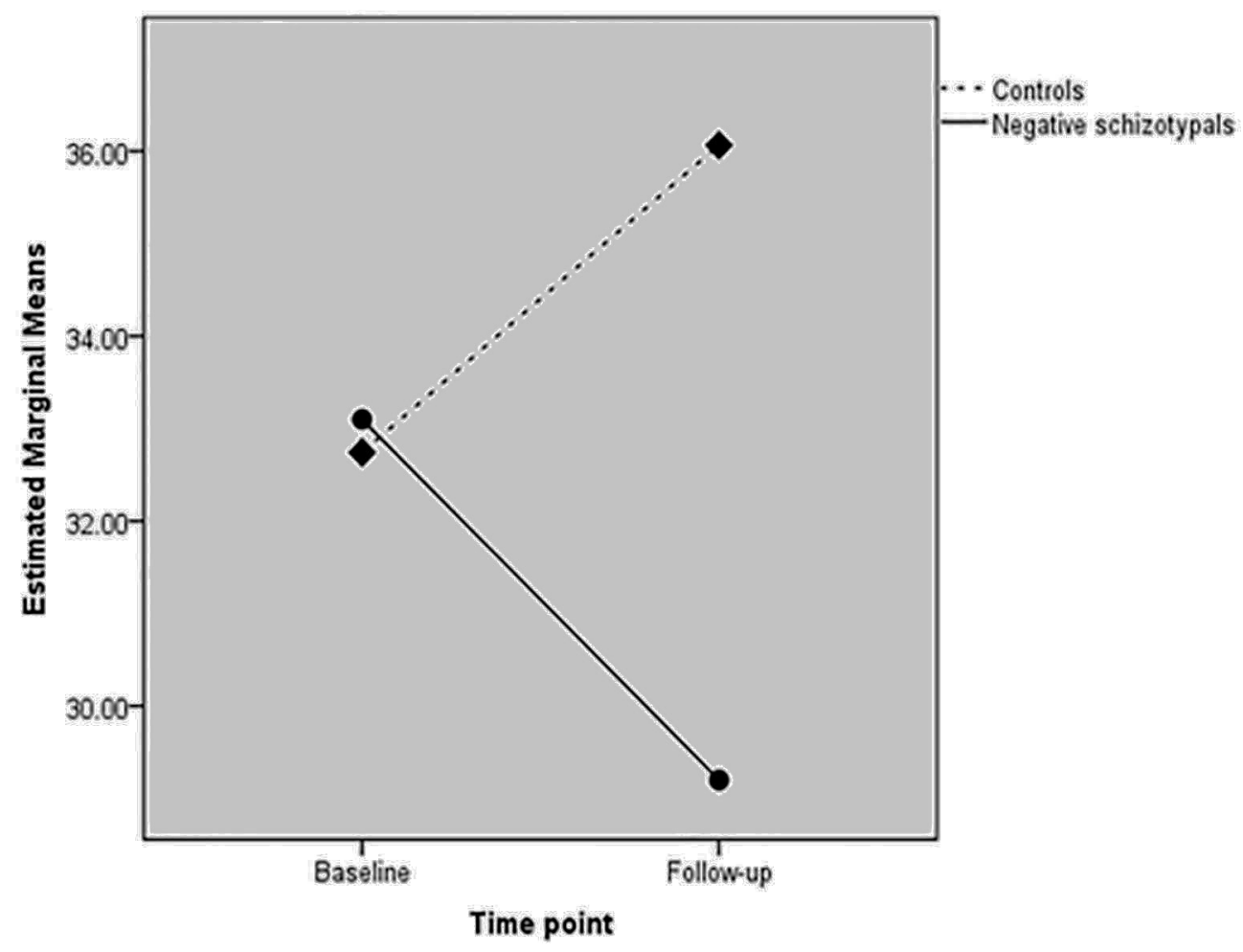

FIGURE 1 | Group $\times$ time point interactions in the unrelated cards of the Wisconsin Card Sorting test (upper panel; A) and switches in semantic verbal fluency (lower panel; B). 
$\left.F_{(1,49)}=6.83, p<0.05 ; \eta^{2}=0.122\right]$. We did not find any other significant main effects or interactions (all $p$-values $>0.095$ ].

\section{Phonemic Verbal Fluency}

Significant main effects of time point were found for correct responses $\left[F_{(1,49)}=10.76, p<0.005 ; \eta^{2}=0.180\right]$ and number of switches $\left[F_{(1,49)}=13.14, p<0.001 ; \eta^{2}=0.211\right]$ as both groups gave more correct responses and made more switches at followup compared with the baseline assessment. No other significant main effects or interactions were revealed (all $p$-values $>0.062$ ).

\section{Semantic Verbal Fluency}

A significant group $\times$ time point interaction was revealed for the number of switches $\left[F_{(1,49)}=6.20, p<0.05 ; \eta^{2}=0.112\right]$ according to which, the negative schizotypal group made fewer switches at follow-up compared with the baseline assessment, while the control group presented with the opposite pattern (Figure 1, lower panel). We also found a significant main effect of time point for the number of correct responses $\left[F_{(1,49)}=8.67\right.$, $\left.p<0.005 ; \eta^{2}=0.150\right]$ with both groups improving at follow-up. The analyses did not reveal any other significant main effects or interactions (all $p$-values $>0.055$ ).

\section{Attention Switch Task}

A significant main effect of time point was revealed for the total correct switched responses $\left[F_{(1,49)}=6.42, p<0.05 ; \eta^{2}=0.118\right]$, with both groups improving at follow-up. The remaining main effects or interactions were not significant (all $p$-values $>0.106$ ).

\section{DISCUSSION}

\section{Stability of Schizotypal Traits and Neuropsychological Tasks Performance}

As regards schizotypal traits, a different pattern emerged when examining the total sample and the sub-sample of "schizotypy congruent" individuals (i.e., participants who at follow-up fulfilled the criteria of inclusion in the same schizotypal group as in the baseline assessment) separately. Thus, in the total sample we found that schizotypal traits did not remain stable over a 4years period, as a decrease in the scores of all schizotypal factors, SPQ total score and the majority of sub-scale scores was found at follow-up. Contrary to this, in the "schizotypy congruent" subgroup, negative schizotypy (and all its constituents) remained unchanged, as did ideas of reference (which play a central role in paranoid schizotypy), unusual perceptual experiences (which is a core feature of cognitive-perceptual schizotypy) and odd speech (one of the two characteristics of disorganized schizotypy). The decrease in schizotypal traits at follow-up is in accordance with findings indicating fluctuations in SPD-like features $(59,83,84)$ or schizotypal traits per se $(61,65,66)$ over time. Nevertheless, certain schizotypal traits (i.e., the negative schizotypal dimension, ideas of reference, unusual perceptual experiences and odd speech) seem to have a more enduring nature in certain individuals (i.e., those falling in the extreme ends in the continuum of schizotypy). Plausible explanations for these findings are based on the facts that negative schizotypy is the sub-clinical analog of negative symptoms, which have been reported to be a stable feature of schizophrenia (85, 86). Further supporting the association of negative schizotypy with negative symptoms, Cohen et al. (84) recently reported that individuals with increased social anhedonia presented with increased negative symptom characteristics over a 3-years period. With respect to the other schizotypal traits that remained stable, unusual perceptual experiences and ideas of reference have also been reported to show stability over time in SPD (87) as is the case with odd/disorganized speech in schizophrenia (88). The correlational analyses aimed to further explore the rank order stability of participants' scores, i.e., “...how individuals maintain their standing on a trait level compared to others in a population over time" [(89), p. 27]. We found that the rank order stability of negative, paranoid and cognitive-perceptual factor scores ranged from very high to high while disorganized schizotypy was moderately rank-ordered; both groups also had a similar pattern of rank order stability at a sub-scale level. This is the first study to report findings on the rank order stability of schizotypal traits with the four-factor model and it is especially interesting that the reported findings are in accordance with the schizophrenia literature indicating high rank order stability of negative symptoms (90) as well as findings suggesting that aspects of paranoid and cognitive-perceptual schizotypy are the most prevalent and highly rank ordered features of SPD (87).

In accordance with previous studies that have employed the same neuropsychological tasks as in the present one and have examined the stability of cognitive functioning (91-96), we found that there was no difference in planning/problem solving, spatial working memory/strategy formation, processing speed, executive working memory and abstract reasoning between the two time points. On the other hand, response inhibition and aspects of cued attention switching, set-shifting as well as phonemic and semantic verbal fluency improved at follow-up compared with baseline, although the effect of time on set-shifting was abolished in the "schizotypy congruent" sub-group probably due to the smaller sample size. Even though there are findings supporting the stability of these cognitive functions over time (93, 9799), there is also evidence indicating that performance in the tasks employed here is subject to practice effects resulting in improved performance (99-103). The majority of these studies include assessments at shorter time-intervals than in the present one; however, there are findings supporting the persistence of these effects at time intervals comparable to the 4-years of our follow-up assessment (104-106).

\section{Prediction of Neuropsychological Performance at Follow-Up by Baseline Schizotypy}

This is the first study to explore the predictive significance of schizotypal traits on cognitive functions after a 4-years period. Moreover, we examined the afore-mentioned associations in our total sample and in the sub-sample of "schizotypy congruent" individuals, separately. It was interesting to find out that a different pattern of associations was revealed between different schizotypal dimensions or specific schizotypal traits in the two groups. 
High negative schizotypy at baseline predicted poorer response inhibition (as examined with more errors in the most "inhibition-demanding" condition of the SST task) and poorer semantic switching (i.e., shifting to another semantic category in order to produce more correct responses) 4 years later both in the total sample and in the "schizotypy congruent" group. Ettinger et al. (107) have reported that negative schizotypy is associated with poor response inhibition cross-sectionally, in analogy to findings showing associations between negative schizophrenia symptoms with both response inhibition (108) and semantic switching (109). The present findings, therefore, suggest that the negative effect of schizotypy on these two executive processes also remains stable over time. Response inhibition and semantic switching are mediated by a frontaltemporal-parietal network in healthy individuals (110-113) and in schizophrenia patients (114-116). Interestingly, alterations within this network seem to be of central importance in SPD as reduced gray matter volume $(117,118)$ has been reported in SPD patients along with associations between cortical thinning (119) or gray matter volume reductions (118) with SPD symptoms. Although the literature on schizotypy is still limited, there is evidence implicating this neural network in negative schizotypy $(120,121)$, as well. Therefore, the critical link between all implicated constructs (i.e., negative schizotypy, response inhibition, semantic switching, schizophrenia, SPD) seems to be the neural circuitry connecting the frontal, temporal, and parietal lobes. Although the methodology of the present study allows only for indirect conclusions, we propose that inefficient functioning of/processing within this network is a persistent feature of negative schizotypy identifying sub-optimal cognitive functioning mediated by this network over time.

High disorganized schizotypy at baseline predicted more intrusion errors and tended to predict fewer correct responses in the semantic fluency task only in the total sample. Tan and Rossell (122) have already reported reduced semantic fluency productivity as disorganized schizotypy increases. In the present study, though, we found a stronger predictive value of disorganized schizotypy for semantic intrusion errors (i.e., production of words belonging to a semantically different category). Correct responses potentially reflect semantic information reserve capacities, while semantic intrusion errors could be considered as failures in semantic processing either due to poor strategic search of representations classified according to their meaning or misattribution of meaning to representations. It is of note, that disorganized schizotypy has been associated with a wide neural network encompassing several brain structures, such as the superior longitudinal fasciculus (121), hippocampus (123), and superior temporal gyrus (124), that are crucial for semantic processing (125-131), thus proving a plausible explanation for our finding. The fact that the association of disorganized schizotypy and semantic processing was abolished in the "schizotypy congruent" sub-group is most probably to the smaller sample size of this group resulting in limited variation of semantic fluency intrusion errors that is required for associational analyses.

High disorganized schizotypy at baseline also predicted poorer complex processing speed/set-shifting, as assessed with the multitasking second part of TMT, both in the total sample and "schizotypy congruent" individuals. In support of this finding, cross-sectional studies have revealed that disorganized schizotypy correlates with response inhibition in relatives of schizophrenia patients as well as controls (107) and disorganization symptoms in schizophrenia patients have been associated with TMT performance $(132,133)$ and other measures of response inhibition (134). At a neuroanatomical level, the neural substrate underlying performance in TMT includes a wide frontal-temporal-parietal network with a frontal cluster including the cingulate and insular cortices and the frontal gyrus acting as a central node (135). Disorganized schizotypy has been associated with reduced volume of the insula (124), cingulate cortex and frontal gyrus $(121,124)$ as well as altered insular information processing (136), and thinning of the anterior cingulate (137). Taken together, our findings suggest that the association of disorganized schizotypy with cognitive functioning relies on different mechanisms and holds out over time: a frontaltemporal network mediates the relationship of disorganized schizotypy with semantic processing while a cluster of frontal regions is the key-neural substratum for connecting disorganized schizotypy with complex processing speed/set-shifting.

\section{Differences in Neuropsychological Tasks Performance Between Negative Schizotypals and Controls}

The follow-up assessment did not include participants falling in all schizotypal dimensions due to the high-rate of drop-out; thus, between-group differences were limited between negative schizotypals and controls and due the between-group differences in most schizotypal dimensions, we could assume that the former group has an overall "heavier dose" of schizotypal traits compared with the latter. As regards the effects of time and in accordance with the analyses of the total sample, neuropsychological task performance in both groups improved at follow-up in measures of response inhibition, set-shifting, phonemic and semantic fluency and cued attention switching. The analyses also revealed that the group of negative schizotypals had poorer response inhibition (as assessed with the SST), set-shifting (as assessed with the completed categories in the WCST), and complex processing speed/set-shifting (examined with the second part of TMT) compared with controls at both time points. These findings (a) cannot be attributed to differences in the demographic characteristics of the participants as there were no betweengroup differences in these variables, (b) are in accordance with previous cross-sectional studies $(69,107,138)$, (c) highlight the persistent nature of specific inefficiencies in cognition in negative schizotypy, and (d) supplement the association of this schizotypal dimension with processes underlaid by the frontal-temporalparietal network described in the previous section.

Interesting group $\times$ time point interactions were also revealed in two fundamental measures: the negative schizotypal group was more prone to failure to maintain set (139) as indicated by the selection of more unrelated cards in the WCST (i.e., choosing a card that has no common features with the target stimuli after acquiring the sorting principle) and made fewer semantic 
switches at follow-up compared with baseline performance; the opposite pattern was observed in controls. Maintaining set and switching are inter-related as they both require efficient self-monitoring, response inhibition and vigilance. They are also primary features of successful performance in the WCST and semantic fluency tasks [e.g., reported correlations between failure to maintain set and completed categories as well as perseverative errors (140) and between semantic switching and word production $(141,142)]$. With respect to the present findings, it is tempting to propose that maintaining set and semantic switching in negative schizotypals are progressively deteriorating thus leading to increased effort for the successful completion of the tasks. These findings, however, are preliminary and the aforementioned suggestion should be viewed with greater caution as far as maintaining set is concerned due to the reported low test-retest reliability of this measure $(143,144)$.

\section{Conclusions}

Taken together, the findings of the present study indicate that schizotypal traits, when analyzed with a detailed four-factor model, decrease over a 4-years period in the general population. The exception is negative schizotypy and odd speech, which is a central feature of disorganized schizotypy; these traits remained stable, in accordance to the literature on schizophrenia and spectrum disorders. The stability of cognitive functioning also varied over time. Thus, response inhibition, aspects of cued attention switching, set-shifting as well as phonemic and semantic verbal fluency are more likely to improve, possibly due to persistent learning/practice effects when performing the same test twice. The need for alternate forms of neuropsychological tasks has already been highlighted $(145,146)$ and the present study suggests that this applies not only in clinical but also in research settings.

High negative schizotypy at baseline predicted poorer response inhibition and semantic switching at follow-up, further supporting and supplementing the involvement of a frontaltemporal-parietal network in all these latent constructs. High disorganized schizotypy at baseline predicted poorer semantic processing and complex processing speed/set-shifting. A frontaltemporal network is suggested to mediate the association of disorganized schizotypy with semantic processing while a cluster of frontal regions is suggested as the key-element for its connection with complex processing speed/set-shifting. The association of negative schizotypy with cognition over time was further explored with preliminary findings on differences between negative schizotypal and control individuals. Poor response inhibition, set-shifting, and complex processing speed/set-shifting were found to be consistently impaired in the former group, as they performed lower than controls in both time points. The ability to maintain set and semantic switching, though, were found to be progressively deteriorating in negative schizotypy. The present findings highlight the importance of taking into consideration the differences in schizotypal traits when designing early-intervention programs for high-risk populations. The fact that different association patterns with cognition were revealed, further advances the formulation of more targeted approaches depending on the prevailing schizotypal traits not only in the schizophrenia spectrum but also in other clusters of mental disorders; for example features of negative schizotypy have been associated with aspects (147-149) or the prevalence rates (5) of mood disorders.

\section{Strengths and Limitations of the Study}

To our knowledge, this is the first study exploring associations of schizotypal traits with cognitive functioning over a 4-years period. It is of note that the participants were community residents covering a wide age-range instead of the most commonly included college students' samples that have been reported to under-represent the general population (150). For the assessment of schizotypy we applied the detailed fourfactor model, which allows for more thorough delineations of schizotypal traits. We also applied strict normative criteria for the identification of negative schizotypal and control individuals instead of dividing the participants according to a samplewise approach (e.g., by median or percentile splits in the current sample).

Nevertheless, there are certain limitations that should be taken into account. First, we had a quite high drop-out rate in the initial sample resulting in a small sample size at follow-up that also differed in demographics compared with the drop-out participants. This also resulted in a small number of "schizotypy incongruent" individuals that were not examined at all at followup. Second, we assessed schizotypy only with a self-report scale, which is widely used but was recently reported not to be fully concordant with interview-based assessments (151). Third, although we examined the subjective state of mood and feelings on the day of testing, we did not include these data in our regression analyses (this would increase the complexity of our models to a level that could not be justified by the current sample sizes) and we did not examine other factors (e.g., anxiety or discontentment on a daily basis) that might have interfered with the participants' performance, especially since the study was conducted during the COVID-19 pandemic.

\section{DATA AVAILABILITY STATEMENT}

The raw data supporting the conclusions of this article will be made available by the authors, without undue reservation.

\section{ETHICS STATEMENT}

The studies involving human participants were reviewed and approved by the Research Ethics Committee of the University of Crete. The patients/participants provided their written informed consent to participate in this study.

\section{AUTHOR CONTRIBUTIONS}

PK collected the data, conducted the initial analyses, and wrote the first draft of the manuscript. CZ collected the data and supplemented the manuscript. SG designed the study and supplemented the statistical analyses and the manuscript. 
All authors contributed to the article and approved the submitted version.

\section{FUNDING}

PK was supported by the Hellenic Foundation for Research and Innovation (HFRI) under the HFRI PhD Fellowship grant (Fellowship Number: 986). CANTAB was acquired with a Start-Up grant by the Special Account for Research of the University of Crete (KA 3748).

\section{REFERENCES}

1. Meehl PE. Schizotaxia revisited. Arch Gen Psychiatry. (1989) 46:935-44. doi: 10.1001/archpsyc.1989.01810100077015

2. Claridge G, Beech T. Fully and quasi-dimensional constructions of schizotypy. In: Raine A, Lencz T, Mednick SA, editors. Schizotypal Personality. Cambridge: Cambridge University Press (1995). p. 192-216.

3. Lenzenweger M, Korfine L. Tracking the taxon: on the latent structure and base rate of schizotypy. In: Raine A, Lencz T, Mednick AA, editors. Schizotypal Personality. Cambridge: Cambridge University Press (1995). p. 135-67.

4. Tyrka AR, Cannon TD, Haslam N, Mednick SA, Schulsinger F, Schulsinger $\mathrm{H}$, et al. The latent structure of schizotypy: I. Premorbid indicators of a taxon of individuals at risk for schizophrenia-spectrum disorders. J Abnorm Psychol. (1995) 104:173-83. doi: 10.1037//0021-843x.104. 1.173

5. Blanchard JJ, Collins LM, Aghevli M, Leung WW, Cohen AS. Social anhedonia and schizotypy in a community sample: the Maryland Longitudinal Study of Schizotypy. Schizophr Bull. (2011) 37:587-602. doi: 10.1093/schbul/sbp107

6. Kwapil TR, Gross GM, Silvia PJ, Barrantes-Vidal N. Prediction of psychopathology and functional impairment by positive and negative schizotypy in the Chapmans' Ten-Year Longitudinal Study. J Abnorm Psychol. (2013) 122:807-15. doi: 10.1037/a0033759

7. Racioppi A, Sheinbaum T, Gross GM, Ballespí S, Kwapil TR, Barrantes-Vidal N. Prediction of prodromal symptoms and schizophrenia-spectrum personality disorder traits by positive and negative schizotypy: a 3-year prospective study. PLoS ONE. (2018) 13:e0207150. doi: 10.1371/journal.pone.02 07150

8. Barkus E, Stirling J, French P, Morrison A, Bentall R, Lewis S. Distress and metacognition in psychosis prone individuals: comparing high schizotypy to the at-risk mental state. J Nerv Ment Dis. (2010) 198:99-104. doi: 10.1097/NMD.0b013e3181cc418a

9. Boldrini T, Tanzilli A, Pontillo M, Chirumbolo A, Vicari S, Lingiardi V. Comorbid personality disorders in individuals with an at-risk mental state for psychosis: a meta-analytic review. Front Psychiatry. (2019) 10:429. doi: 10.3389/fpsyt.2019.00429

10. Giakoumaki SG. Cognitive and prepulse inhibition deficits in psychometrically high schizotypal subjects in the general population: relevance to schizophrenia research. J Int Neuropsychol Soc. (2012) 18:643-56. doi: 10.1017/S135561771200029X

11. Giakoumaki SG. Emotion processing deficits in the different dimensions of psychometric schizotypy. Scand J Psychol. (2016) 57:256-70. doi: 10.1111/sjop.12287

12. Nelson MT, Seal ML, Pantelis C, Phillips LJ. Evidence of a dimensional relationship between schizotypy and schizophrenia: a systematic review. Neurosci Biobehav Rev. (2013) 37:317-27. doi: 10.1016/j.neubiorev.2013.01.004

13. Ettinger U, Meyhöfer I, Steffens M, Wagner $M$, Koutsouleris $N$. Genetics, cognition, and neurobiology of schizotypal personality: a review of the overlap with schizophrenia. Front Psychiatry. (2014) 5:18. doi: $10.3389 /$ fpsyt.2014.00018

\section{ACKNOWLEDGMENTS}

The authors wish to thank the participants for their help with the study.

\section{SUPPLEMENTARY MATERIAL}

The Supplementary Material for this article can be found online at: https://www.frontiersin.org/articles/10.3389/fpsyt. 2020.613015/full\#supplementary-material

14. Ettinger U, Mohr C, Gooding DC, Cohen AS, Rapp A, Haenschel C, et al. Cognition and brain function in schizotypy: a selective review. Schizophr Bull. (2015) 41:S417-26. doi: 10.1093/schbul/sbu190

15. Rosell DR, Futterman SE, McMaster A, Siever LJ. Schizotypal personality disorder: a current review. Curr Psychiatry Rep. (2014) 16:452. doi: 10.1007/s11920-014-0452-1

16. Barrantes-Vidal N, Grant P, Kwapil TR. The role of schizotypy in the study of the etiology of schizophrenia spectrum disorders. Schizophr Bull. (2015) 41:S408-416. doi: 10.1093/schbul/sbu191

17. Modenato C, Draganski B. The concept of schizotypy-a computational anatomy perspective. Schizophr Res Cogn. (2015) 2:89-92. doi: 10.1016/j.scog.2015.05.001

18. Debbané M, Eliez S, Badoud D, Conus P, Flückiger R, Schultze-Lutter F. Developing psychosis and its risk states through the lens of schizotypy. Schizophr Bull. (2015) 41:S396-407. doi: 10.1093/schbul/sbu176

19. Flückiger R, Ruhrmann S, Debbané M, Michel C, Hubl D, Schimmelmann $\mathrm{BG}$, et al. Psychosis-predictive value of self-reported schizotypy in a clinical high-risk sample. J Abnorm Psychol. (2016) 125:923-32. doi: 10.1037/abn0000192

20. Zarogianni E, Storkey AJ, Johnstone EC, Owens DGC, Lawrie SM. Improved individualized prediction of schizophrenia in subjects at familial high risk, based on neuroanatomical data, schizotypal and neurocognitive features. Schizophr Res. (2017) 181:6-12. doi: 10.1016/j.schres.2016.08.027

21. Bang M, Park JY, Kim KR, Lee SY, Song YY, Kang JI, et al. Psychotic conversion of individuals at ultra-high risk for psychosis: the potential roles of schizotypy and basic symptoms. Early Interv Psychiatry. (2019) 13:546-54. doi: 10.1111/eip.12518

22. Kotlicka-Antczak M, Karbownik MS, Pawełczyk A, Zurner N, Pawełczyk T, Strzelecki D, et al. A developmentally-stable pattern of premorbid schizoid-schizotypal features predicts psychotic transition from the clinical high-risk for psychosis state. Compr Psychiatry. (2019) 90:95-101. doi: 10.1016/j.comppsych.2019.02.003

23. Dazzan P, Arango C, Fleischacker W, Galderisi S, Glenthøj B, Leucht S, et al. Magnetic resonance imaging and the prediction of outcome in firstepisode schizophrenia: a review of current evidence and directions for future research. Schizophr Bull. (2015) 41:574-83. doi: 10.1093/schbul/sbv024

24. Keshavan MS, Collin G, Guimond S, Kelly S, Prasad KM, Lizano P. Neuroimaging in schizophrenia. Neuroimaging Clin N Am. (2020) 30:73-83. doi: 10.1016/j.nic.2019.09.007

25. Giegling I, Hosak L, Mössner R, Serretti A, Bellivier F, Claes S, et al. Genetics of schizophrenia: a consensus paper of the WFSBP task force on genetics. World J Biol Psychiatry. (2017) 18:492-505. doi: 10.1080/15622975.2016.1268715

26. Prata DP, Costa-Neves B, Cosme G, Vassos E. Unravelling the genetic basis of schizophrenia and bipolar disorder with GWAS: a systematic review. J Psychiatr Res. (2019) 114:178-207. doi: 10.1016/j.jpsychires.2019.04.007

27. DiLalla LF, McCrary M, Diaz E. A review of endophenotypes in schizophrenia and autism: the next phase for understanding genetic etiologies. Am J Med Genet C Semin Med Genet. (2017) 175:354-61. doi: 10.1002/ajmg.c.31566

28. Khan A, Powell SB. Sensorimotor gating deficits in "Two-Hit" models of schizophrenia risk factors. Schizophr Res. (2018) 198:68-83. doi: 10.1016/j.schres.2017.10.009 
29. Szabo S, Merikle E, Lozano-Ortega G, Powell L, Macek T, Cline S. Assessing the relationship between performance on the University of California Performance Skills Assessment (UPSA) and outcomes in schizophrenia: a systematic review and evidence synthesis. Schizophr Res Treat. (2018) 27:9075174. doi: 10.1155/2018/9075174

30. Chan SKW, Chan HYV, Devlin J, Bastiampillai T, Mohan T, Hui CLM, et al. A systematic review of long-term outcomes of patients with psychosis who received early intervention services. Int Rev Psychiatry. (2019) 31:425-40. doi: 10.1080/09540261.2019.1643704

31. Bora E, Akdede BB, Alptekin K. Neurocognitive impairment in deficit and non-deficit schizophrenia: a meta-analysis. Psychol Med. (2017) 47:2401-13. doi: $10.1017 /$ S0033291717000952

32. Thai ML, Andreassen AK, Bliksted V. A meta-analysis of executive dysfunction in patients with schizophrenia: different degree of impairment in the ecological subdomains of the Behavioural Assessment of the Dysexecutive Syndrome. Psychiatry Res. (2019) 272:230-6. doi: 10.1016/j.psychres.2018.12.088

33. Kelly S, Guimond S, Lyall A, Stone WS, Shenton ME, Keshavan M, et al. Neural correlates of cognitive deficits across developmental phases of schizophrenia. Neurobiol Dis. (2019) 131:104353. doi: 10.1016/j.nbd.2018.12.013

34. Penadés R, Franck N, González-Vallespí L, Dekerle M. Neuroimaging studies of cognitive function in schizophrenia. Adv Exp Med Biol. (2019) 1118:11734. doi: 10.1007/978-3-030-05542-4_6

35. Keefe, R. S. (2014). The longitudinal course of cognitive impairment in schizophrenia: an examination of data from premorbid through posttreatment phases of illness. J. Clin. Psychiatry. 75, S8-13. doi: 10.4088/JCP.13065su1.02

36. Mollon J, Reichenberg A. Cognitive development prior to onset of psychosis. Psychol Med. (2018) 48:392-403. doi: 10.1017/S0033291717001970

37. Bora E, Pantelis C. Meta-analysis of cognitive impairment in first-episode bipolar disorder: comparison with first-episode schizophrenia and healthy controls. Schizophr Bull. (2015) 4:1095-104. doi: 10.1093/schbul/sbu198

38. Bora E, Lin A, Wood SJ, Yung AR, McGorry PD, Pantelis C. Cognitive deficits in youth with familial and clinical high risk to psychosis: a systematic review and meta-analysis. Acta Psychiatr Scand. (2014) 130:1-15. doi: 10.1111 /acps.12261

39. Zheng W, Zhang QE, Cai DB, Ng CH, Ungvari GS, Ning YP, et al. Neurocognitive dysfunction in subjects at clinical high risk for psychosis: a meta-analysis. J Psychiatr Res. (2018) 103:38-45. doi: 10.1016/j.jpsychires.2018.05.001

40. Agnew-Blais J, Seidman LJ. Neurocognition in youth and young adults under age 30 at familial risk for schizophrenia: a quantitative and qualitative review. Cogn Neuropsychiatry. (2013) 18:44-82. doi: 10.1080/13546805.2012.676309

41. Siddi S, Petretto DR, Preti A. Neuropsychological correlates of schizotypy: a systematic review and meta-analysis of cross-sectional studies. Cogn Neuropsychiatry. (2017) 22:186-212. doi: 10.1080/13546805.2017.1299702

42. Park S, Holzman PS, Lenzenweger MF. Individual differences in spatial working memory in relation to schizotypy. J Abnorm Psychol. (1995) 104:355-63. doi: 10.1037//0021-843x.104.2.355

43. Aguirre F, Sergi MJ, Levy CA. Emotional intelligence and social functioning in persons with schizotypy. Schizophr Res. (2008) 104:255-64. doi: 10.1016/j.schres.2008.05.007

44. Hori H, Matsuo J, Teraishi T, Sasayama D. Schizotypy and genetic loading for schizophrenia impact upon neuropsychological status in bipolar II and unipolar major depressive disorders. J Affect Disord. (2012) 142:225-32. doi: 10.1016/j.jad.2012.04.031

45. Koychev I, El-Deredy W, Haenschel C, Deakin JFW. Visual information processing deficits as biomarkers of vulnerability to schizophrenia: an eventrelated potential study in schizotypy. Neuropsychologia. (2010) 48:2205-14. doi: 10.1016/j.neuropsychologia.2010.04.014

46. Suhr JA. Executive functioning deficits in hypothetically psychosis-prone college students. Schizophr Res. (1997) 27:29-35. doi: 10.1016/s0920-9964(97)00072-8

47. Kim MS, Oh SH, Hong MH, Choi DB. Neuropsychologic profile of college students with schizotypal traits. Compr Psychiatry. (2011) 52:511-6. doi: $10.1016 /$ j. comppsych.2010.10.010
48. Lenzenweger MF, Gold JM. Auditory working memory and verbal recall memory in schizotypy. Schizophr Res. (2000) 42:101-10. doi: 10.1016/s0920-9964(99)00121-8

49. Jahshan CS, Sergi MJ. Theory of mind, neurocognition, and functional status in schizotypy. Schizophr Res. (2007) 89:278-86. doi: 10.1016/j.schres.2006.09.004

50. Noguchi H, Hori H, Kunugi H. Schizotypal traits and cognitive function in healthy adults. Psychiatry Res. (2008) 161:162-9. doi: 10.1016/j.psychres.2007.07.023

51. Spitznagel MB, Suhr JA. Executive function deficits associated with symptoms of schizotypy and obsessive-compulsive disorder. Psychiatry Res. (2002) 110:151-63. doi: 10.1016/s0165-1781(02)00099-9

52. Cimino M, Haywood M. Inhibition and facilitation in schizotypy. J Clin Exp Neuropsychol. (2008) 30:187-98. doi: 10.1080/13803390701336866

53. Sheffield JM, Karcher NR, Barch DM. Cognitive deficits in psychotic disorders: a lifespan perspective. Neuropsychol Rev. (2018) 28:509-33. doi: $10.1007 /$ s11065-018-9388-2

54. McCleery A, Nuechterlein KH. Cognitive impairment in psychotic illness: prevalence, profile of impairment, developmental course, and treatment considerations. Dialog Clin Neurosci. (2019) 21:239-48. doi: 10.31887/DCNS.2019.21.3/amccleery

55. Chan RCK, Shi H, Geng F, Liu W, Yan C, Wang Y, et al. The Chapman psychosis-proneness scales: consistency across culture and time. Psychiatry Res. (2015) 228:143-9. doi: 10.1016/j.psychres.2015.04.031

56. Raine A. The SPQ: a scale for the assessment of schizotypal personality disorder based on DSM-III-R criteria. Schizophr Bull. (1991) 17:555-64. doi: 10.1093/schbul/17.4.555

57. Ericson M, Tuvblad C, Raine A, Young-Wolff K, Baker LA. Heritability and longitudinal stability of schizotypal traits during adolescence. Behav Genet. (2011) 41:499-511. doi: 10.1007/s10519-010-9401-x

58. Venables PH, Raine A. The stability of schizotypy across time and instruments. Psychiatry Res. (2015) 228:585-90. doi: 10.1016/j.psychres.2015.05.047

59. Geng FL, Xu T, Wang Y, Shi HS, Yan C, Neumann DL, et al. Developmental trajectories of schizotypal personality disorder-like behavioural manifestations: a two-year longitudinal prospective study of college students. BMC Psychiatry. (2013) 13:323. doi: 10.1186/1471-244X-13-323

60. Stefanis NC, Vitoratou S, Ntzouufras J, Smyrnis N, Evdokimisdis J, Stefanis CN. Psychometric properties of the greek version of the Schizotypal Personality Questionnaire (SPQ) in young male obligatory conscripts: a two year test-retest study. Pers Individ Dif. (2006) 41:1275-86. doi: 10.1016/j.paid.2006.07.003

61. Moreno-Izco L, Sánchez-Torres AM, Lorente-Omeñaca R, Fañanás L, Rosa A, Salvatore P, et al. Ten-year stability of self-reported schizotypal personality features in patients with psychosis and their healthy siblings. Psychiatry Res. (2015) 227:283-9. doi: 10.1016/j.psychres.2015.02.020

62. Chapman LJ, Chapman JP, Raulin ML. Scales for physical and social anhedonia. J Abnorm Psychol. (1976) 85:374-82. doi: $10.1037 / / 0021-843 \times .85 .4 .374$

63. Chapman LJ, Chapman JP, Raulin ML. Body-image aberration in schizophrenia. J Abnorm Psychol. (1978) 87:399-407. doi: 10.1037//0021-843x.87.4.399

64. Eckblad M, Chapman LJ. Magical ideation as an indicator of schizotypy. J Consult Clin Psychol. (1983) 51:215-25. doi: 10.1037//0022-006x.51.2.215

65. Wang Y, Shi HS, Liu WH, Xie DJ, Geng FL, Yan C, et al. Trajectories of schizotypy and their emotional and social functioning: an 18-month followup study. Schizophr Res. (2018) 193:384-90. doi: 10.1016/j.schres.2017.07.038

66. Meyer TD, Hautzinger M. Two-year stability of psychosis proneness scales and their relations to personality disorder traits. J Pers Assess. (1999) 73:47288. doi: 10.1207/S15327752JPA7303_11

67. Wang Y, Chan RCK, Cui JF, Yang TX, Deng YY, Gong QY, et al. Stability of prospective memory deficits in individuals with schizotypal personality traits. Psychiatry Res. (2011) 189:156-7. doi: 10.1016/j.psychres.2011.01.002

68. Cohen AS, Couture SM, Blanchard JJ. Neuropsychological functioning and social anhedonia: three-year follow-up data from a longitudinal community high risk study. J Psychiatr Res. (2012) 46:898-904. doi: 10.1016/j.jpsychires.2012.03.020 
69. Karagiannopoulou L, Karamaouna P, Zouraraki C, Roussos P, Bitsios P, Giakoumaki SG. Cognitive profiles of schizotypal dimensions in a community cohort: common properties of differential manifestations. J Clin Exp Neuropsychol. (2016) 38:1050-63. doi: 10.1080/13803395.2016.1188890

70. Tsaousis I, Zouraraki C, Karamaouna P, Karagiannopoulou L, Giakoumaki SG. The validity of the Schizotypal Personality Questionnaire in a Greek sample: tests of measurement invariance and latent mean differences. Compr Psychiatry. (2015) 62:51-62. doi: 10.1016/j.comppsych.2015.06.003

71. Nelson HE. A modified card sorting test sensitive to frontal lobe defects. Cortex. (1976) 12:313-24. doi: 10.1016/s0010-9452(76)80035-4

72. Milner B. Effects of different brain lesions on card sorting. Arch Neurol. (1963) 9:90-100. doi: 10.1001/archneur.1963.00460070100010

73. Wechsler D. Wechsler Adult Intelligence Scale-Fourth Edition (WAIS-IV). San Antonio, TX: NCS Pearson Inc. (2008).

74. Stogiannidou A. Wechsler Adult Intelligence Scale, Standardization in Greek. 4th ed. (WAIS-IV GR). Athens: Motibo (2014).

75. Tombaugh TN. Trail Making Test A and B: normative data stratified by age and education. Arch Clin Neuropsychol. (2004) 19:203-14. doi: 10.1016/S0887-6177(03)00039-8

76. Zalonis I, Kararizou E, Triantafyllou NI, Kapaki E, Papageorgiou $\mathrm{S}$, Sgouropoulos $\mathrm{P}$, et al. A normative study of the trail making test A and B in Greek adults. Clin Neuropsychol. (2008) 22:842-50. doi: 10.1080/13854040701629301

77. Kosmidis MH, Vlahou CH, Panagiotaki P, Kiosseoglou G. The verbal fluency task in the Greek population: normative data, and clustering and switching strategies. J Int Neuropsychol Soc. (2004) 10:164-72. doi: $10.1017 /$ S1355617704102014

78. Raven J, Raven JC, Court JH. Manual for Raven's Progressive Matrices and Vocabulary Scales. Section 1: General Overview. San Antonio, TX: Harcourt Assessment (2003).

79. Robbins TW, James M, Owen AM, Sahakian BJ, Lawrence AD, McInnes L, et al. A study of performance on tests from the CANTAB battery sensitive to frontal lobe dysfunction in a large sample of normal volunteers: implications for theories of executive functioning and cognitive aging. J Int Neuropsychol Soc. (1998) 4:474-90. doi: 10.1017/S1355617798455073

80. Owen AM, Downes JJ, Sahakian BJ, Polkey CE, Robbins TW. Planning and spatial working memory following frontal lobe lesions in man. Neuropsychologia. (1990) 28:1021-34. doi: 10.1016/0028-3932(90)90137-D

81. Norris $H$. The action of sedatives on brain stem oculomotor systems in man. Neuropharmacology. (1971) 10:181-91. doi: 10.1016/0028-3908(71) 90039-6

82. Bond $\mathrm{AJ}$, Lader $\mathrm{MH}$. The use of analogue scales in rating subjective feelings. Br J Med Psychol. (1974) 47:211-8. doi: 10.1111/j.2044-8341.1974.tb02285.x

83. Grilo CM, Sanislow CA, Gunderson JG, Pagano ME, Yen S, Zanarini MC, et al. Two-year stability and change of schizotypal, borderline, avoidant, and obsessive-compulsive personality disorders. J Consult Clin Psychol. (2004) 72:767-75. doi: 10.1037/0022-006X.72.5.767

84. Cohen AS, Couture SM, Blanchard JJ. Social anhedonia and clinical outcomes in early adulthood: a three-year follow-up study within a community sample. Schizophr Res. (2020) 10:S0920-9964(20)30417-5. doi: 10.1016/j.schres.2020.07.024

85. Mezquida G, Cabrera B, Bioque M, Amoretti S, Lobo A, González-Pinto A, et al. The course of negative symptoms in first-episode schizophrenia and its predictors: a prospective two-year follow-up study. Schizophr Res. (2017) 189:84-90. doi: 10.1016/j.schres.2017.01.047

86. Haro JM, Altamura C, Corral R, Elkis H, Evans J, Krebs MO, et al. Understanding the course of persistent symptoms in schizophrenia: longitudinal findings from the pattern study. Psychiatry Res. (2018) 267:5662. doi: 10.1016/j.psychres.2018.04.005

87. McGlashan MH, Grilo CM, Sanislow CA, Ralevski E, Morey LC, Gunderson JG, et al. Two-year prevalence and stability of individual DSM-IV criteria for schizotypal, borderline, avoidant, and obsessive-compulsive personality disorders: toward a hybrid model of axis II disorders. Am J Psychiatry. (2005) 162:883-9. doi: 10.1176/appi.ajp.162.5.883

88. Marengo J, Harrow M, Herbener ES, Sands J. A prospective longitudinal 10year study of schizophrenia's three major factors and depression. Psychiatry Res. (2000) 97:61-77. doi: 10.1016/s0165-1781(00)00218-3
89. Hamlat EJ, Young JF, Hankin BL. Developmental course of personality disorder traits in childhood and adolescence. J Pers Disord. 34:25-43. doi: 10.1521/pedi_2019_33_433

90. Reichenberg A, Rieckmann N, Harvey PD. Stability in schizophrenia symptoms over time: findings from the Mount Sinai Pilgrim Psychiatric Center Longitudinal Study. J Abnorm Psychol. (2005) 114:363-72. doi: 10.1037/0021-843X.114.3.363

91. Raven J. The Raven's progressive matrices: change and stability over culture and time. Cogn Psychol. (2000) 41:1-48. doi: 10.1006/cogp.1999.0735

92. Tyson PJ, Laws KR, Roberts KH, Mortimer AM. Stability of set-shifting and planning abilities in patients with schizophrenia. Psychiatry Res. (2004) 129:229-39. doi: 10.1016/j.psychres.2004.09.007

93. Ornstein TJ, Sahakian BJ, McKenna PJ. Memory and executive impairment in schizophrenia: comparison with frontal and temporal brain damage. Psychol Med. (2008) 38:833-42. doi: 10.1017/S0033291707001468

94. Light GA, Swerdlow NR, Rissling AJ, Radant A, Sugar CA, Sprock J, et al. Characterization of neurophysiologic and neurocognitive biomarkers for use in genomic and clinical outcome studies of schizophrenia. PLoS ONE. (2012) 7:e39434. doi: 10.1371/journal.pone.0039434

95. Juuhl-Langseth M, Holmén A, Thormodsen R, Oie M, Rund BR. Relative stability of neurocognitive deficits in early onset schizophrenia spectrum patients. Schizophr Res. (2014) 156:241-7. doi: 10.1016/j.schres.2014.04.014

96. Daglas R, Allott K, Yücel M, Pantelis C, Macneil CA, Berk M, et al. The trajectory of cognitive functioning following first episode mania: a 12-month follow-up study. Aust N Z J Psychiatry. (2016) 50:1186-97. doi: $10.1177 / 0004867415622272$

97. Sánchez-Torres AM, Basterra V, Moreno-Izco L, Rosa A, Fañanás L, Zarzuela A, et al. Executive functioning in schizophrenia spectrum disorder patients and their unaffected siblings: a ten-year follow-up study. Schizophr Res. (2013) 143:291-6. doi: 10.1016/j.schres.2012.11.026

98. Wöstmann NM, Aichert DS, Costa A, Rubia K, Möller HJ, Ettinger U. Reliability and plasticity of response inhibition and interference control. Brain Cogn. (2013) 81:82-94. doi: 10.1016/j.bandc.2012.09.010

99. Strobach T, Wendt M, Tomat M, Luna-Rodriguez A, Jacobsen T. No evidence for the reduction of task competition and attentional adjustment during task-switching practice. Acta Psychol (Amst). (2020) 204:103036. doi: 10.1016/j.actpsy.2020.103036

100. Basso MR, Lowery N, Ghormley C, Bornstein RA. Practice effects on the Wisconsin card sorting test-64 card version across 12 months. Clin Neuropsychol. (2001) 15:471-8. doi: 10.1076/clin.15.4.471.1883

101. Ross TP. The reliability of cluster and switch scores for the controlled oral word association test. Arch Clin Neuropsychol. (2003) 18:153-64. doi: 10.1016/S0887-6177(01)00192-5

102. Palmer CE, Langbehn D, Tabrizi SJ, Papoutsi M. Test-retest reliability of measures commonly used to measure striatal dysfunction across multiple testing sessions: a longitudinal study. Front Psychol. (2018) 8:2363. doi: 10.3389/fpsyg.2017.02363

103. Karlsen RH, Karr JE, Saksvik SB, Lundervold AJ, Hjemdal O, Olsen A, et al. Examining 3-month test-retest reliability and reliable change using the Cambridge Neuropsychological Test Automated Battery. Appl Neuropsychol Adult. (2020) 21:1-9. doi: 10.1080/23279095.2020.1722126

104. Rabbitt P, Diggle P, Holland F, McInnes L. Practice and drop-out effects during a 17-year longitudinal study of cognitive aging. J Gerontol B Psychol Sci Soc Sci. (2004) 59:84-97. doi: 10.1093/geronb/59.2.p84

105. Rabbitt P, Lunn M, Wong D, Cobain M. Age and ability affect practice gains in longitudinal studies of cognitive change. J Gerontol B Psychol Sci Soc Sci. (2008) 63:235-40. doi: 10.1093/geronb/63.4.p235

106. Salthouse TA. Effects of age and ability on components of cognitive change. Intelligence. (2013) 41:501-11. doi: 10.1016/j.intell.2013.07.005

107. Ettinger U, Aichert DS, Wöstmann N, Dehning S, Riedel M, Kumari V. Response inhibition and interference control: effects of schizophrenia, genetic risk, and schizotypy. J Neuropsychol. (2018) 12:484-510. doi: 10.1111/jnp.12126

108. Shin YS, Kim SN, Shin NY, Jung WH, Hur JW, Byun MS, et al. Increased intra-individual variability of cognitive processing in subjects at risk mental state and schizophrenia patients. PLoS ONE. (2013) 8:e78354. doi: 10.1371/journal.pone.0078354 
109. Egeland J, Holmen TL, Bang-Kittilsen G, Bigseth TT, Engh JA. Category fluency in schizophrenia: opposing effects of negative and positive symptoms? Cogn Neuropsychiatry. (2018) 23:28-42. doi: 10.1080/13546805.2017.1418306

110. Birn RM, Kenworthy L, Case L, Caravella R, Jones TB, Bandettini PA, et al. Neural systems supporting lexical search guided by letter and semantic category cues: a self-paced overt response fMRI study of verbal fluency. Neuroimage. (2010) 49:1099-107. doi: 10.1016/j.neuroimage.2009.07.036

111. Hu S, Li CS. Neural processes of preparatory control for stop signal inhibition. Hum Brain Mapp. (2012) 33:2785-96. doi: 10.1002/hbm.21399

112. Scheuringer A, Harris TA, Pletzer B. Recruiting the right hemisphere: Sex differences in inter-hemispheric communication during semantic verbal fluency. Brain Lang. (2020) 207:104814. doi: 10.1016/j.bandl.2020.104814

113. Wang B, Hao Y, Zhan Q, Zhao S, Li D, Imtiaz S, et al. Dynamic reconfiguration of functional brain networks supporting response inhibition in a stop-signal task. Brain Imaging Behav. (2020) 14:2500-11. doi: 10.1007/s11682-019-00203-7

114. Ragland JD, Moelter ST, Bhati MT, Valdez JN, Kohler CG, Siegel SJ, et al. Effect of retrieval effort and switching demand on fMRI activation during semantic word generation in schizophrenia. Schizophr Res. (2008) 99:312-23. doi: 10.1016/j.schres.2007.11.017

115. Naim-Feil J, Rubinson M, Freche D, Grinshpoon A, Peled A, Moses E, et al. Altered brain network dynamics in schizophrenia: a cognitive electroencephalography study. Biol Psychiatry Cogn Neurosci Neuroimaging. (2018) 3:88-98. doi: 10.1016/j.bpsc.2017.03.017

116. Tsujii N, Mikawa W, Adachi T, Hirose T, Shirakawa O. Shared and differential cortical functional abnormalities associated with inhibitory control in patients with schizophrenia and bipolar disorder. Sci Rep. (2018) 8:4686. doi: 10.1038/s41598-018-22929-y

117. Koo MS, Dickey CC, Park HJ, Kubicki M, Ji NY, Bouix S, et al. Smaller neocortical gray matter and larger sulcal cerebrospinal fluid volumes in neuroleptic-naive women with schizotypal personality disorder. Arch Gen Psychiatry. (2006) 63:1090-100. doi: 10.1001/archpsyc.63.10.1090

118. Asami T, Whitford TJ, Bouix S, Dickey CC, Niznikiewicz M, Shenton ME, et al. Globally and locally reduced MRI gray matter volumes in neuroleptic-naive men with schizotypal personality disorder: association with negative symptoms. JAMA Psychiatry. (2013) 70:361-72. doi: 10.1001/jamapsychiatry.2013.665

119. Watsky RE, Pollard KL, Greenstein D, Shora L, Dillard-Broadnax D, Gochman P, et al. Severity of cortical thinning correlates with schizophrenia spectrum symptoms. J Am Acad Child Adolesc Psychiatry. (2016) 55:130-6. doi: 10.1016/j.jaac.2015.11.008

120. Kühn S, Schubert F, Gallinat J. Higher prefrontal cortical thickness in high schizotypal personality trait. J Psychiatr Res. (2012) 46:960-5. doi: 10.1016/j.jpsychires.2012.04.007

121. Pfarr JK, Nenadić I. A multimodal imaging study of brain structural correlates of schizotypy dimensions using the MSS. Psychiatry Res Neuroimaging. (2020) 302:111104. doi: 10.1016/j.pscychresns.2020.111104

122. Tan EJ, Rossell SL. Disorganised schizotypy is selectively associated with poorer semantic processing in non-clinical individuals. Psychiatry Res. (2017) 256:249-52. doi: 10.1016/j.psychres.2017.06.067

123. Sahakyan L, Meller T, Evermann U, Schmitt S, Pfarr JK, Sommer $\mathrm{J}$, et al. Anterior vs posterior hippocampal subfields in an extended psychosis phenotype of multidimensional schizotypy in a nonclinical sample. Schizophr Bull. (2020) 21:sbaa099. doi: 10.1093/schbul/sbaa099

124. Wiebels K, Waldie KE, Roberts RP, Park HR. Identifying grey matter changes in schizotypy using partial least squares correlation. Cortex. (2016) 81:13750. doi: 10.1016/j.cortex.2016.04.011

125. Meijer JH, Schmitz N, Nieman DH, Becker HE, van Amelsvoort TA, Dingemans PM, et al. Semantic fluency deficits and reduced grey matter before transition to psychosis: a voxelwise correlational analysis. Psychiatry Res. (2011) 194:1-6. doi: 10.1016/j.pscychresns.2011.01.004

126. Rodríguez-Aranda C, Waterloo K, Johnsen SH, Eldevik P, Sparr S, Wikran GC, et al. Neuroanatomical correlates of verbal fluency in early Alzheimer's disease and normal aging. Brain Lang. (2016) 155-156:24-35. doi: 10.1016/j.bandl.2016.03.001

127. Li M, Zhang Y, Song L, Huang R, Ding J, Fang Y, et al. Structural connectivity subserving verbal fluency revealed by lesion-behavior mapping in stroke patients. Neuropsychologia. (2017) 101:85-96. doi: 10.1016/j.neuropsychologia.2017.05.008

128. Briggs RG, Chakraborty AR, Anderson CD, Abraham CJ, Palejwala AH, Conner AK, et al. Anatomy and white matter connections of the inferior frontal gyrus. Clin Anat. (2019) 32:546-56. doi: 10.1002/ca.23349

129. Manca R, Stabile MR, Bevilacqua F, Cadorin C, Piccione F, Sharrack $\mathrm{B}$, et al. Cognitive speed and white matter integrity in secondary progressive multiple sclerosis. Mult Scler Relat Disord. (2019) 30:198-207. doi: 10.1016/j.msard.2019.02.021

130. Schmidt CSM, Nitschke K, Bormann T, Römer P, Kümmerer D, Martin $\mathrm{M}$, et al. Dissociating frontal and temporal correlates of phonological and semantic fluency in a large sample of left hemisphere stroke patients. Neuroimage Clin. (2019) 23:101840. doi: 10.1016/j.nicl.2019.101840

131. Paek EJ, Murray LL, Newman SD. Neural correlates of verb fluency performance in cognitively healthy older adults and individuals with dementia: a pilot fMRI study. Front Aging Neurosci. (2020) 12:73. doi: $10.3389 /$ fnagi.2020.00073

132. Brazo P, Marié RM, Halbecq I, Benali K, Segard L, Delamillieure P, et al. Cognitive patterns in subtypes of schizophrenia. Eur Psychiatry. (2002) 17:155-62. doi: 10.1016/s0924-9338(02)00648-x

133. Galderisi S, Davidson M, Kahn RS, Mucci A, Boter H, Gheorghe $\mathrm{MD}$, et al. Correlates of cognitive impairment in first episode schizophrenia: the EUFEST study. Schizophr Res. (2009) 115:104-14. doi: 10.1016/j.schres.2009.09.022

134. Woodward TS, Ruff CC, Thornton AE, Moritz S, Liddle PF. Methodological considerations regarding the association of Stroop and verbal fluency performance with the symptoms of schizophrenia. Schizophr Res. (2003) 61:207-14. doi: 10.1016/s0920-9964(02) 00211-6

135. Varjacic A, Mantini D, Demeyere N, Gillebert CR. Neural signatures of trail making test performance: evidence from lesion-mapping and neuroimaging studies. Neuropsychologia. (2018) 115:78-87. doi: 10.1016/j.neuropsychologia.2018.03.031

136. Ford TC, Woods W, Crewther DP. Spatio-temporal source cluster analysis reveals fronto-temporal auditory change processing differences within a shared autistic and schizotypal trait phenotype. Neuroimage Clin. (2017) 25:383-9. doi: 10.1016/j.nicl.2017.04.022

137. Derome M, Tonini E, Zöller D, Schaer M, Eliez S, Debbané M. Developmental trajectories of cortical thickness in relation to schizotypy during adolescence. Schizophr Bull. (2020) 5:sbaa020. doi: $10.1093 / \mathrm{schbul} / \mathrm{sbaa} 020$

138. Laurent A, Duly D, Murry P, Foussard N, Boccara S, Mingat F, et al. WCST performance and schizotypal features in the first-degree relatives of patients with schizophrenia. Psychiatry Res. (2001) 104:133-44. doi: 10.1016/s0165-1781(01)00306-7

139. Figueroa IJ, Youmans RJ. Failure to maintain set: a measure of distractibility or cognitive flexibility? Proc Hum Factors Ergon Soc Annu Meet. (2013) 57:828-32. doi: 10.1177/1541931213571180

140. Banno M, Koide T, Aleksic B, Okada T, Kikuchi T, Kohmura $\mathrm{K}$, et al. Wisconsin card sorting test scores and clinical and sociodemographic correlates in schizophrenia: multiple logistic regression analysis. BMJ Open. (2012) 2:6. doi: 10.1136/bmjopen-2012001340

141. Cralidis A, Lundgren K. Component analysis of verbal fluency performance in younger participants with moderate-to-severe traumatic brain injury. Brain Inj. (2014) 28:456-64. doi: 10.3109/02699052.2014.896945

142. Scheuringer A, Wittig R, Pletzer B. Sex differences in verbal fluency: the role of strategies and instructions. Cogn Process. (2017) 18:407-17. doi: 10.1007/s10339-017-0801-1

143. Chiu EC, Lee SC. Test-retest reliability of the Wisconsin card sorting test in people with schizophrenia. Disabil Rehabil. (2019) 30:1-5. doi: 10.1080/09638288.2019.1647295

144. Kopp B, Lange F, Steinke A. The reliability of the Wisconsin card sorting test in clinical practice. Assessment. (2019) 2:1073191119866257. doi: $10.1177 / 1073191119866257$

145. Duff K. Evidence-based indicators of neuropsychological change in the individual patient: relevant concepts and methods. Arch Clin Neuropsychol. (2012) 27:248-61. doi: 10.1093/arclin/acr120 
146. Calamia M, Markon K, Tranel D. The robust reliability of neuropsychological measures: meta-analyses of test-retest correlations. Clin Neuropsychol. (2013) 27:1077-105. doi: 10.1080/13854046.2013.809795

147. Watson D, Naragon-Gainey K. On the specificity of positive emotional dysfunction in psychopathology: evidence from the mood and anxiety disorders and schizophrenia/schizotypy. Clin Psychol Rev. (2010) 30:839-48. doi: 10.1016/j.cpr.2009.11.002

148. Martin EA, Becker TM, Cicero DC, Docherty AR, Kerns JG. Differential associations between schizotypy facets and emotion traits. Psychiatry Res. (2011) 187:94-9. doi: 10.1016/j.psychres.2010.12.028

149. Kemp KC, Gross GM, Barrantes-Vidal N, Kwapil TR. Association of positive, negative, and disorganized schizotypy dimensions with affective symptoms and experiences. Psychiatry Res. (2018) 270:1143-9. doi: 10.1016/j.psychres.2018.10.031

150. Chun CA, Minor KS, Cohen AS. Neurocognition in psychometrically defined college schizotypy samples: we are not measuring the "right stuff". J Int Neuropsychol Soc. (2013) 19:324-37. doi: 10.1017/S135561771200152X
151. Chan CC, Bulbena-Cabre A, Rutter S, Benavides C, McClure MM Calabrese W, et al. Comparison of self-report and clinician-rated schizotypal traits in schizotypal personality disorder and community controls. Schizophr Res. (2019) 209:263-8. doi: 10.1016/j.schres.2018. 12.050

Conflict of Interest: The authors declare that the research was conducted in the absence of any commercial or financial relationships that could be construed as a potential conflict of interest.

Copyright (c) 2021 Karamaouna, Zouraraki and Giakoumaki. This is an open-access article distributed under the terms of the Creative Commons Attribution License (CC BY). The use, distribution or reproduction in other forums is permitted, provided the original author(s) and the copyright owner(s) are credited and that the original publication in this journal is cited, in accordance with accepted academic practice. No use, distribution or reproduction is permitted which does not comply with these terms. 